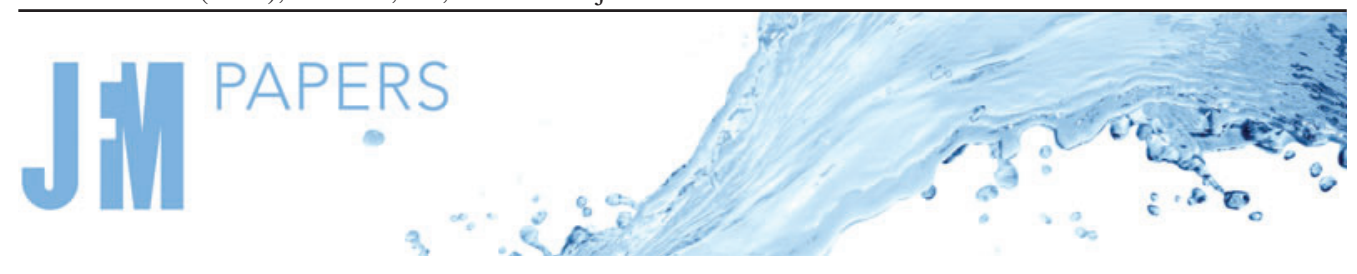

\title{
Three-dimensionality of hypersonic laminar flow over a double cone
}

\author{
Jiaao Hao ${ }^{1}$, Jianhui Fan ${ }^{1}$, Shibin $\mathrm{Cao}^{1}$ and Chih-Yung Wen ${ }^{1,} \dagger$ \\ ${ }^{1}$ Department of Aeronautical and Aviation Engineering, The Hong Kong Polytechnic University, \\ Kowloon, Hong Kong
}

(Received 19 July 2021; revised 1 December 2021; accepted 14 December 2021)

\begin{abstract}
Hypersonic laminar flow over a canonical $25^{\circ}-55^{\circ}$ double cone is studied using computational fluid dynamics and global stability analysis (GSA) with a free-stream Mach number of 11.5 and various unit Reynolds numbers. Axisymmetric simulations reveal that secondary separation occurs beneath the primary separation bubble beyond a critical Reynolds number. The numerical results agree well with existing experiments and the triple-deck theory with the axisymmetric effect on the incoming boundary layer treated by the Mangler transformation. The GSA identifies a three-dimensional global instability that is azimuthally periodic immediately prior to the emergence of secondary separation. The criterion of the onset of global instability in terms of a scaled deflection angle established for supersonic compression corner flows (Hao et al., J. Fluid Mech., vol. 919, 2021, A4) can be directly applied to double-cone flows. As the Reynolds number is further increased, the flow is strongly destabilized with the coexistence of multiple stationary and low-frequency oscillating unstable modes. Direct numerical simulations confirm that the supercritical double-cone flow is intrinsically three-dimensional, unsteady and exhibits strong azimuthal variations in the peak heating.
\end{abstract}

Key words: high-speed flow, boundary layer separation, hypersonic flow

\section{Introduction}

Hypersonic flow over a double cone represents a canonical case of shock-wave-boundarylayer interaction (Babinsky \& Harvey 2011). A double cone can sustain a strong interaction phenomenon and obviate the finite-span effects of its two-dimensional counterpart (i.e. a double wedge). Therefore, hypersonic double-cone flows have been extensively studied

† Email address for correspondence: cywen@polyu.edu.hk

(C) The Author(s), 2022. Published by Cambridge University Press. This is an Open Access article, distributed under the terms of the Creative Commons Attribution licence (https://creativecommons. org/licenses/by/4.0/), which permits unrestricted re-use, distribution, and reproduction in any medium, provided the original work is properly cited. 
to understand shock-induced separation and validate computational fluid dynamics (CFD) tools.

A series of $25^{\circ}-55^{\circ}$ double-cone experiments has been conducted at Calspan-University of Buffalo Research Center over the past two decades with a wide range of free-stream Mach numbers, Reynolds numbers and total enthalpies in the LENS I reflected shock tunnel and LENS XX expansion tunnel (Holden \& Wadhams 2003; Holden et al. 2003; Holden et al. 2015). Surface pressure and heat flux distributions were measured. Various numerical simulations have been performed and compared with the experiments (Gaitonde, Canupp \& Holden 2002; Nompelis, Candler \& Holden 2003; Druguet, Candler \& Nompelis 2005; Knight et al. 2012; Hao \& Wen 2018a,b). However, these CFD validation campaigns were only a partial success. Under high-enthalpy conditions with evident vibrational excitation and chemical reactions, the CFD significantly underestimated the size of the separation region. In contrast, good agreement was usually obtained at low enthalpies. However, the axisymmetric simulations predicted a dramatically unsteady flow in some circumstances, whereas the experiments suggested the opposite (Knight et al. 2012). Currently, these discrepancies are still not understood.

Historically, flow unsteadiness in shock-wave-laminar boundary-layer interactions has received much less attention than turbulent ones. In hypersonic laminar flow over a double cone, unsteadiness may occur due to different physical mechanisms. Hornung, Gollan \& Jacobs (2021) numerically studied axisymmetric double-cone flows with varying flow parameters. Pulsating unsteadiness was found to be an inviscid phenomenon consistent with the pulsation mode in supersonic spiked-body flows. In particular, the parameter space of the first and second cone angles was explored to determine the unsteadiness boundary. The lower boundary decreased to approach the shock detachment angle of the second cone as the first cone angle was decreased. Tumuklu, Theofilis \& Levin (2018) performed time-accurate direct Monte Carlo simulations of Mach 16 flows over the $25^{\circ}-55^{\circ}$ double cone with different unit Reynolds numbers. For the highest-Reynolds-number case, the Kelvin-Helmholtz instability arising in the shear layer and a bow-shock oscillation were captured with dominant frequencies of 45-70 and $2 \mathrm{kHz}$, respectively. Interestingly, the residuals algorithm identified a temporally stable axisymmetric global mode, highlighting a strong coupling between the separation region and shock system. Compared with the well-known pulsation mode and Kelvin-Helmholtz instability of an inviscid nature, the onset of global instability intrinsic to double-cone flows merits further investigation.

Nominally two-dimensional/axisymmetric laminar separation bubbles can sustain three-dimensional instabilities (Theofilis, Hein \& Dallmann 2000; Theofilis 2011) with no upstream disturbances, which have been studied by the global stability analysis (GSA) approach. In the supersonic flow regime, global instabilities are further complicated by compressibility and complex shock structures. Robinet (2007) observed low-frequency unsteady characteristics of shock impingement on a Mach 2.15 laminar boundary layer using direct numerical simulations (DNS), which stemmed from a supercritical Hopf bifurcation of the separated flow. Global stability analysis confirmed that the flow became globally unstable beyond a critical angle of the incident shock. Similarly, Hildebrand et al. (2018) studied shock impingement on a Mach 5.92 laminar boundary layer. They found a stationary global mode at a supercritical shock angle, which can lead to the formation of streamwise streaks downstream of reattachment.

For a Mach 5 double-wedge flow, Sidharth et al. (2018) found a stationary unstable mode beyond a critical deflection angle, which was attributed to the streamwise deceleration of the separated flow. At a supercritical deflection angle, multiple stationary and oscillating unstable modes were captured. Cao et al. (2021) observed streamwise streaks in heat flux 


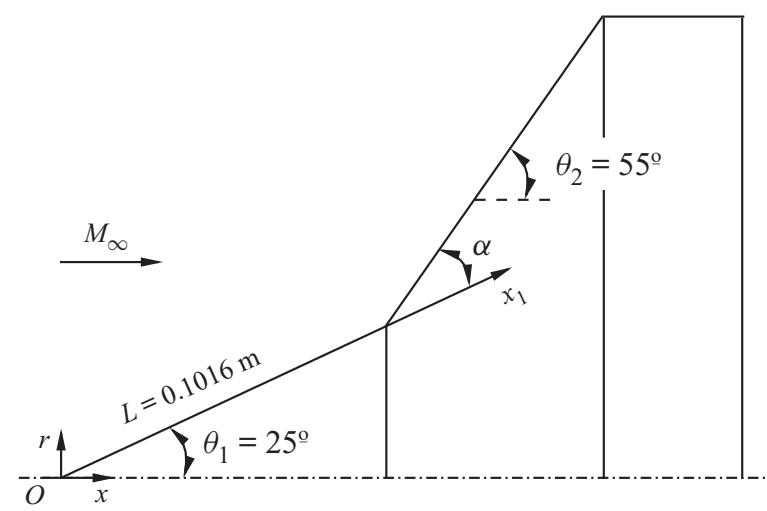

Figure 1. Schematic of the double-cone configuration.

on the ramp surface in hypersonic flow over a $15^{\circ}$ compression corner with low-frequency unsteadiness using DNS. The two-dimensional base flow was found to be globally unstable by GSA, which identified one stationary and two oscillating modes. Good agreement was obtained between DNS and GSA in terms of the spanwise wavelengths of the streaks and dominant frequencies. More recently, the global stability of a Mach 7.7 compression corner flow was parametrically studied by Hao et al. (2021) with different ramp angles and wall temperatures, which was closely linked with the emergence of secondary separation beneath the primary bubble. As the ramp angle was increased beyond a certain value, a long-wavelength stationary unstable mode first occurred, followed by a short-wavelength stationary mode and additional oscillating modes. Furthermore, it was found that the stability boundary can be well correlated in terms of a scaled ramp angle according to the triple-deck theory (Neiland 1969; Stewartson \& Williams 1969).

In previous GSA studies, only two-dimensional flow problems were considered, except that of Tumuklu et al. (2018). However, their residuals analysis was restricted to axisymmetric scenarios with only temporally stable modes. The bifurcation of axisymmetric double-cone flows into three-dimensionality remains to be understood. To this end, hypersonic flow over the $25^{\circ}-55^{\circ}$ double-cone configuration is investigated numerically and theoretically in this study. Axisymmetric CFD and GSA are performed over a range of unit Reynolds numbers. The base flows are compared with existing experimental data and interpreted using the triple-deck theory. The corresponding linear stability spectra are then explored by assuming periodic perturbations in the azimuthal direction. Finally, DNS are performed to verify the GSA results and reveal the evolution of three-dimensional flow structures.

\section{Geometric configuration and flow conditions}

The experimental model tested in the LENS I and LENS XX tunnels is considered here, as shown in figure 1 . The $25^{\circ}-55^{\circ}$ double cone has a sharp nose with two equal-length conical sections $(L=0.1016 \mathrm{~m})$. A cylindrical coordinate system is constructed with the origin at the nose, the $x$ direction along the cone axis, the $r$ direction perpendicular to the axis and the $\phi$ direction satisfying the right-hand rule.

The baseline flow conditions are taken from run 35 (Holden et al. 2003) with free-stream conditions $M_{\infty}=11.5, \rho_{\infty}=5.515 \times 10^{-4} \mathrm{~kg} \mathrm{~m}^{-3}, T_{\infty}=138.9 \mathrm{~K}, u_{\infty}=2713 \mathrm{~m} \mathrm{~s}^{-1}$ and $R e_{\infty}=1.6 \times 10^{5} \mathrm{~m}^{-1}$. Due to the low total enthalpy, the air is assumed to be calorically perfect with a constant specific heat ratio of 1.4. In fact, a certain amount of vibrational 
energy was frozen in the nozzle exit of the LENS I reflected shock tunnel. However, as shown by Nompelis et al. (2003), the inclusion of the vibrational energy and its weak accommodation at the wall only improved the predictive accuracy of the heat flux on the first cone. Therefore, no vibrational energy is considered in this study. The flow-field variables are non-dimensionalized using the free-stream parameters. The length of the conical section $L$ is used as the characteristic length of the flow.

Three additional flow conditions are considered, with the free-stream density $\rho_{\infty}$ decreased from the baseline value by a factor of $0.75,0.5$ and 0.33 , while the other free-stream quantities remain unchanged. For convenience, these cases are labelled $0.75 R e$, $0.5 R e$ and $0.33 R e$.

According to Hornung et al. (2021), the pulsation mode is enclosed in the lower and upper boundaries of the second cone angle $\left(\theta_{2}\right)$ for a given first cone angle $\left(\theta_{1}\right)$. The lower boundary is located above the shock detachment angle $\left(\theta_{d}\right)$ of the second cone with respect to $M_{\infty}$. For the current flow conditions, $\theta_{d}$ is approximately $57^{\circ}$ as determined from the Taylor-Maccoll equations, which indicates that the considered $25^{\circ}-55^{\circ}$ double-cone flows sustain no inviscid unsteadiness.

\section{Computational details}

\subsection{Governing equations}

The governing equations are the compressible Navier-Stokes equations for a calorically perfect gas written in the following conservation form:

$$
\frac{\partial \boldsymbol{U}}{\partial t}+\frac{\partial \boldsymbol{F}}{\partial x}+\frac{1}{r} \frac{\partial(r \boldsymbol{G})}{\partial r}+\frac{1}{r} \frac{\partial \boldsymbol{H}}{\partial \phi}=\frac{1}{r} \boldsymbol{Q},
$$

where $U=[\rho, \rho u, \rho v, \rho w, \rho E]^{\mathrm{T}}$ is the vector of conservative variables and the vectors of fluxes and source terms are given by

$$
\begin{aligned}
& \boldsymbol{F}=\left[\begin{array}{c}
\rho u \\
\rho u^{2}+p \\
\rho v u \\
\rho w u \\
(\rho E+p) u
\end{array}\right]+\left[\begin{array}{c}
0 \\
\tau_{x x} \\
\tau_{r x} \\
\tau_{\phi x} \\
u \tau_{x x}+v \tau_{r x}+w \tau_{\phi x}+q_{x}
\end{array}\right], \\
& \boldsymbol{G}=\left[\begin{array}{c}
\rho v \\
\rho u v \\
\rho v^{2}+p \\
\rho w v \\
(\rho E+p) v
\end{array}\right]+\left[\begin{array}{c}
0 \\
\tau_{x r} \\
\tau_{r r} \\
\tau_{\phi r} \\
u \tau_{x r}+v \tau_{r r}+w \tau_{\phi r}+q_{r}
\end{array}\right], \\
& H=\left[\begin{array}{c}
\rho w \\
\rho u w \\
\rho v w \\
\rho w^{2}+p \\
(\rho E+p) w
\end{array}\right]+\left[\begin{array}{c}
0 \\
\tau_{x \phi} \\
\tau_{r \phi} \\
\tau_{\phi \phi} \\
u \tau_{x \phi}+v \tau_{r \phi}+w \tau_{\phi \phi}+q_{\phi}
\end{array}\right] \text {, } \\
& Q=\left[\begin{array}{c}
0 \\
0 \\
p+\rho w^{2}+\tau_{\phi \phi} \\
-\rho v w-\tau_{r \phi} \\
0
\end{array}\right]
\end{aligned}
$$


In these expressions, $\rho$ is the density, $u, v$ and $w$ are the flow velocities in the $x, r$ and $\phi$ directions, respectively, $p$ is the pressure and $E$ is the total energy per unit mass. The shear stresses are calculated according to a Newtonian fluid and the Stokes hypothesis as

$$
\begin{gathered}
\tau_{x x}=-\mu\left[2 \frac{\partial u}{\partial x}-\frac{2}{3}\left(\frac{\partial u}{\partial x}+\frac{\partial v}{\partial r}+\frac{1}{r} \frac{\partial w}{\partial \phi}+\frac{v}{r}\right)\right] \\
\tau_{r r}=-\mu\left[2 \frac{\partial v}{\partial r}-\frac{2}{3}\left(\frac{\partial u}{\partial x}+\frac{\partial v}{\partial r}+\frac{1}{r} \frac{\partial w}{\partial \phi}+\frac{v}{r}\right)\right] \\
\tau_{\phi \phi}=-\mu\left[2\left(\frac{1}{r} \frac{\partial w}{\partial \phi}+\frac{v}{r}\right)-\frac{2}{3}\left(\frac{\partial u}{\partial x}+\frac{\partial v}{\partial r}+\frac{1}{r} \frac{\partial w}{\partial \phi}+\frac{v}{r}\right)\right], \\
\tau_{x r}=\tau_{r x}=-\mu\left(\frac{\partial u}{\partial r}+\frac{\partial v}{\partial x}\right) \\
\tau_{x \phi}=\tau_{\phi x}=-\mu\left(\frac{1}{r} \frac{\partial u}{\partial \phi}+\frac{\partial w}{\partial x}\right) \\
\tau_{r \phi}=\tau_{\phi r}=-\mu\left(\frac{1}{r} \frac{\partial v}{\partial \phi}+\frac{\partial w}{\partial r}-\frac{w}{r}\right)
\end{gathered}
$$

where $\mu$ is the dynamic viscosity evaluated using Sutherland's law. The vector of heat conduction is modelled according to Fourier's law as

$$
q_{x}=-k \frac{\partial T}{\partial x}, \quad q_{r}=-k \frac{\partial T}{\partial r}, \quad q_{\phi}=-k \frac{1}{r} \frac{\partial T}{\partial \phi},
$$

where $k$ is the coefficient of thermal conductivity evaluated using a constant Prandtl number of 0.72 .

It is assumed that $U$ can be decomposed into an axisymmetric steady solution and a three-dimensional small-amplitude perturbation as

$$
U(x, r, \phi, t)=\bar{U}(x, r)+U^{\prime}(x, r, \phi, t) .
$$

Hereafter, the overbar and prime denote base-flow and perturbation variables, respectively.

\subsection{Base-flow solver}

The governing equations of the base flow can be readily obtained from (3.1) by assuming

$$
\bar{w}=0, \quad \frac{\bar{\partial}}{\partial \phi}=0 .
$$

The integral form of the axisymmetric governing equations can then be obtained by applying the divergence theorem to a finite-volume cell of unit radian in the azimuthal direction as

$$
\oint_{S}\left(\overline{\boldsymbol{F}} n_{x}+\overline{\boldsymbol{G}} n_{r}\right) \mathrm{d} S=\int_{V} \frac{1}{r} \overline{\boldsymbol{Q}} \mathrm{d} V,
$$

where $n_{x}$ and $n_{r}$ are the components of the unit normal vector of the control surface, $S$ is the area of the cell surface and $V$ is the cell volume. The discrete form of (3.15) can be expressed as

$$
\frac{1}{V} \sum_{f}\left(\overline{\boldsymbol{F}} n_{x}+\overline{\boldsymbol{G}} n_{r}\right)_{f} S_{f}=\frac{1}{r_{c}} \overline{\boldsymbol{Q}}_{c},
$$

where the subscripts $f$ and $c$ denote face- and cell-averaged values, respectively. 


\section{J. Hao, J. Fan, S. Cao and C.-Y. Wen}

The base-flow solutions are obtained using an in-house multiblock parallel finite-volume solver called PHAROS (Hao, Wang \& Lee 2016; Hao \& Wen 2020). The modified Steger-Warming scheme (MacCormack 2014) is used to calculate the inviscid fluxes, which is extended to a second order by the monotone upstream-centred schemes for conservation law reconstruction (van Leer 1979). The viscous fluxes are computed using the second-order central difference. An implicit line relaxation method (Wright, Candler $\&$ Bose 1998) is used for pseudo time stepping.

The boundary conditions are given as follows. Free-stream conditions are applied to the far-field boundary, whereas the outflow boundary conditions are determined using a simple extrapolation. The model surface is assumed to have no slip with a fixed wall temperature $T_{w}=300 \mathrm{~K}$ corresponding to a wall temperature ratio $T_{w} / T_{0}=0.079$, where $T_{0}$ is the free-stream total temperature.

Numerical simulations are run with a constant Courant-Friedrichs-Lewy number of $10^{3}$ until iterative convergence. For all cases, the Euclidean norm of the density residual is reduced by at least ten orders of magnitude, and the distributions of surface quantities no longer change in successive iterations. No self-sustained instabilities are supported by the considered axisymmetric double-cone flows.

\subsection{Global stability analysis}

The governing equations of $U$ ' can be obtained by substituting (3.13) into the governing equations (3.1) and neglecting higher-order terms as

$$
\frac{\partial \boldsymbol{U}^{\prime}}{\partial t}+\frac{\partial \boldsymbol{F}^{\prime}}{\partial x}+\frac{1}{r} \frac{\partial\left(r \boldsymbol{G}^{\prime}\right)}{\partial r}+\frac{1}{r} \frac{\partial \boldsymbol{H}^{\prime}}{\partial \phi}=\frac{1}{r} \boldsymbol{Q}^{\prime} .
$$

The equations are then integrated and discretized on a finite-volume cell of unit radian in the azimuthal direction as

$$
\frac{\partial \boldsymbol{U}^{\prime}{ }_{c}}{\partial t}+\frac{1}{V} \sum_{f}\left(\boldsymbol{F}^{\prime} n_{x}+\boldsymbol{G}^{\prime} n_{r}\right)_{f} S_{f}+\frac{1}{r_{c}} \frac{\partial \boldsymbol{H}^{\prime}{ }_{c}}{\partial \phi}=\frac{1}{r_{c}} Q_{c}^{\prime} .
$$

As with PHAROS, the inviscid fluxes are calculated using the modified Steger-Warming scheme. A central scheme is activated in smooth regions away from discontinuities detected by an improved Ducros shock sensor to reduce numerical dissipation (Hendrickson, Kartha \& Candler 2018). The viscous fluxes are calculated using the second-order central difference.

Vector $U^{\prime}$ is further assumed to be in the following modal form:

$$
U^{\prime}(x, r, \phi, t)=\hat{U}(x, r) \exp \left[-\mathrm{i}\left(\omega_{r}+\mathrm{i} \omega_{i}\right) t+\mathrm{i} m \phi\right],
$$

where $\hat{U}$ is the axisymmetric eigenfunction, $\omega_{r}$ is the angular frequency, $\omega_{i}$ is the growth rate and $m$ is the azimuthal wavenumber. Note that $m$ must be an integer, with $m=0$ representing an axisymmetric mode. Substituting (3.19) into (3.18) results in an eigenvalue problem as

$$
\bar{A} \hat{U}=\left(\omega_{r}+\mathrm{i} \omega_{i}\right) \hat{U},
$$

where $\bar{A}$ is the global matrix composed of Jacobians of the inviscid and viscous fluxes and source terms. The eigenvalue problem is solved using the implicitly restarted Arnoldi method implemented in ARPACK (Sorensen et al. 1996) for a given $m$. 
(a)

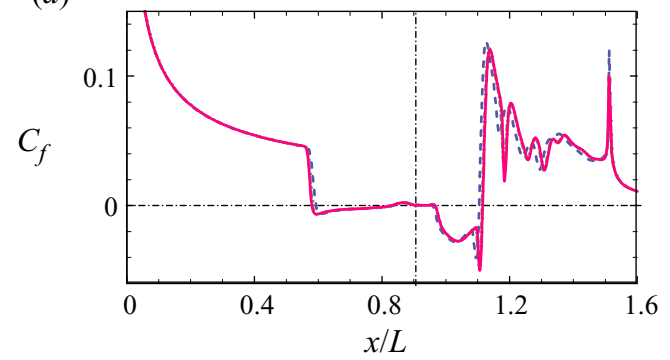

(b)

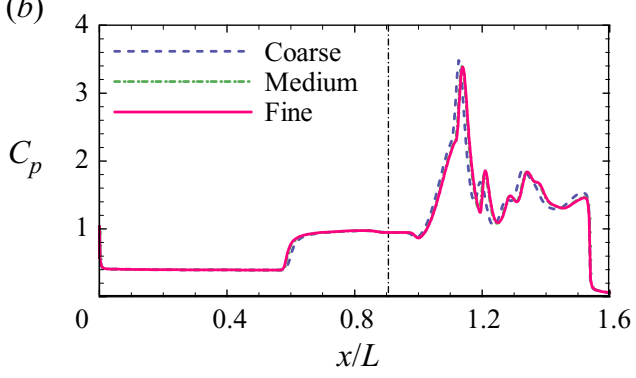

(c)

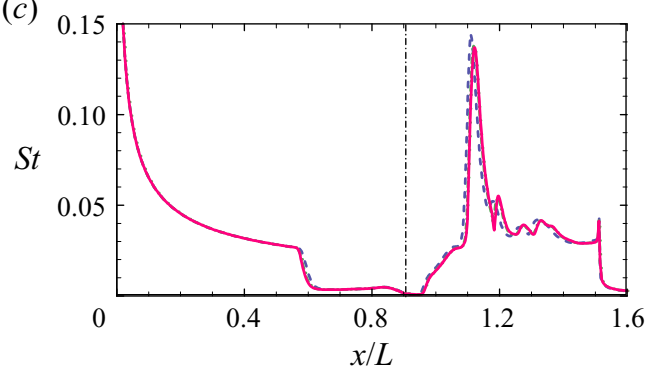

Figure 2. Distributions of (a) skin friction coefficient, $(b)$ surface pressure coefficient and $(c)$ surface Stanton number obtained on three different grids for the baseline case.

At the far-field boundary, all the perturbations are set to zero, which indicates no upstream disturbances. Simple extrapolation is used at the supersonic outflow boundary. The boundary conditions at the solid wall are given by

$$
\hat{u}=\hat{v}=\hat{w}=\hat{T}=\frac{\partial \hat{p}}{\partial n}=0 .
$$

Furthermore, sponge layers are placed near the far-field and outflow boundaries of the computational domain to ensure no reflection of perturbations (Mani 2012).

\subsection{Grid independence study}

Computational grids are constructed with three levels of resolution, namely $500 \times 250$ (coarse), $750 \times 350$ (medium) and $1000 \times 450$ (fine), in the streamwise and wall-normal directions. The normal spacing at the wall is set to $1 \times 10^{-7} \mathrm{~m}$ to ensure that the grid Reynolds number has an order of magnitude of one. Axisymmetric CFD and GSA are performed on these grids to verify grid independence.

Figure 2 compares the distributions of the skin friction coefficient $C_{f}$, surface pressure coefficient $C_{p}$ and surface Stanton number $S t$ obtained on different grids for the baseline case. Herein, $C_{f}, C_{p}$ and $S t$ are defined by

$$
C_{f}=\frac{\tau_{w}}{0.5 \rho_{\infty} u_{\infty}^{2}}, \quad C_{p}=\frac{p_{w}}{0.5 \rho_{\infty} u_{\infty}^{2}}, \quad S t=\frac{q_{w}}{0.5 \rho_{\infty} u_{\infty}^{3}},
$$

where $\tau_{w}, p_{w}$ and $q_{w}$ are the surface shear stress, pressure and heat flux, respectively. Given that the distributions on the medium and fine grids almost overlap, the medium grid should be adequate for CFD.

Figure 3 compares the eigenvalue spectra obtained at different grids for the baseline case corresponding to the most unstable azimuthal wavenumber $m=33$ (discussed in $\S 4.3$ ). 


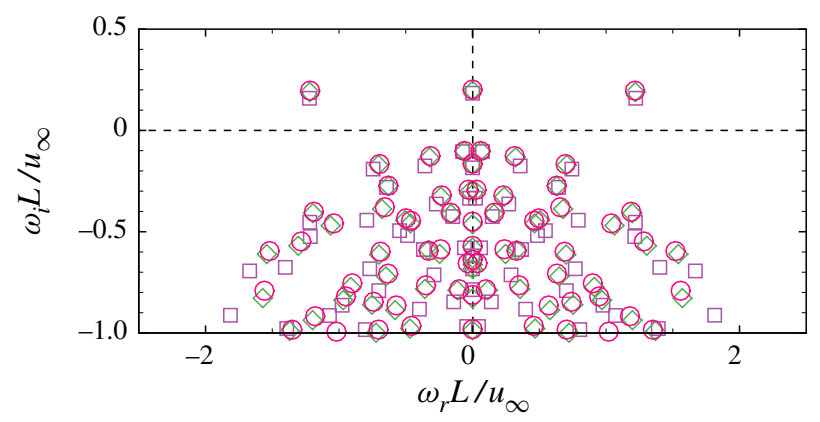

Figure 3. Eigenvalue spectra obtained on three different grids for the baseline case corresponding to the most unstable azimuthal wavenumber $m=33$. Squares, coarse grid; diamonds, medium grid; circles, fine grid.

A stationary and an oscillating unstable mode are captured. Their growth rates increase as the grid is refined. It is indicated that the medium grid is also adequate for GSA.

\section{Results}

\subsection{Base flows}

Figure 4 shows the contours of the density gradient magnitude for different Reynolds numbers superimposed with the sonic lines and streamlines in the separation region. The basic flow structure is largely unaffected by the Reynolds number. An oblique shock is generated by the first cone. If the flow is inviscid, the supersonic flow behind the oblique shock is controlled by the Taylor-Maccoll equations and is deflected into itself again at the corner by the second cone to form another oblique shock. However, due to the complexity of the shock interaction, the oblique shock induced by the second cone is curved such that the postshock flow is locally subsonic (Olejniczak, Wright \& Candler 1997).

If viscosity is accounted for, the adverse pressure gradient at the corner causes the incoming boundary layer to separate. A separation shock and a reattachment shock are further generated by the separation region. The separation shock interacts with the curved shock to form a triple point, a slip line and a transmitted shock. The transmitted and reattachment shocks intersect to form Edney's type I shock interaction (Edney 1968). Downstream of the interaction, the transmitted shock impinges on the second cone, whereas the reattachment shock hits the slip line. As a result, the slip line is deflected to become nearly parallel to the second cone. An underexpanded supersonic jet develops between the slip line and second cone, where the transmitted shock is reflected back and forth to form a series of compression and expansion waves. The streamwise scale of the wave reflection extends downstream as the Reynolds number is increased.

Interestingly, the separation region is always terminated by the impingement of the transmitted shock, which may suggest that the separation region and shock system are strongly coupled. The size of the separation region increases with increasing Reynolds number. For the $0.75 R e$ case, secondary separation occurs and distorts the primary bubble. For the baseline case, the secondary bubble grows, and the primary bubble fragments into two vortices.

Figure 5 displays the distributions of the skin friction coefficient for different Reynolds numbers with enlarged views near the corner. Flow separation and reattachment can be readily determined at the most upstream and downstream locations where the curve of $C_{f}$ crosses zero. Upstream of separation, $C_{f}$ decreases as the laminar boundary layer 
(a)

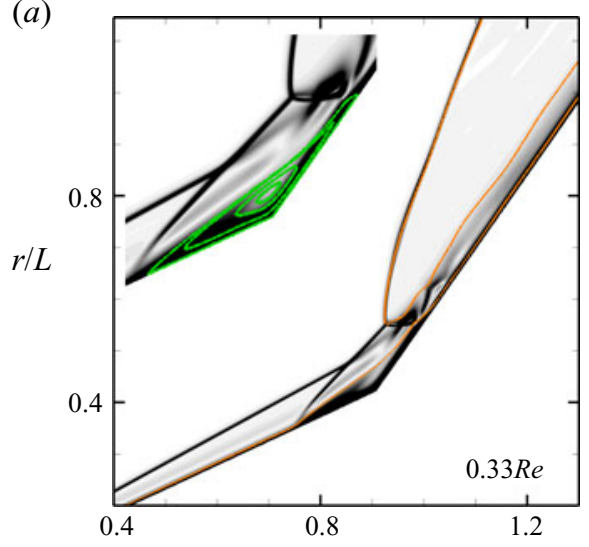

(c)

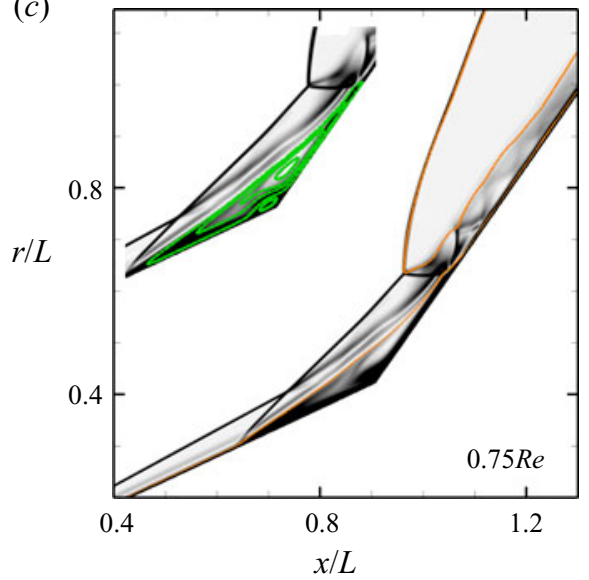

(b)

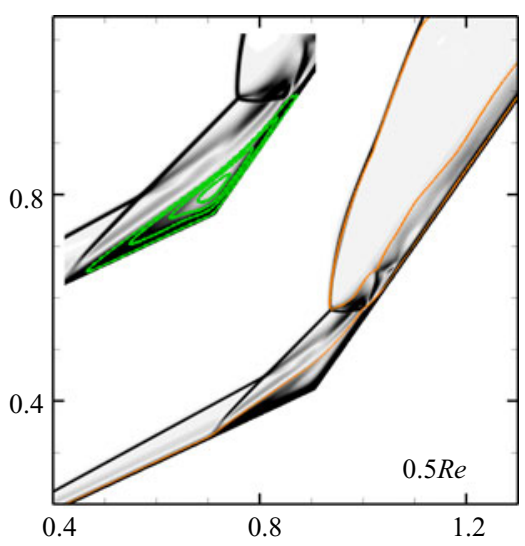

$(d)$

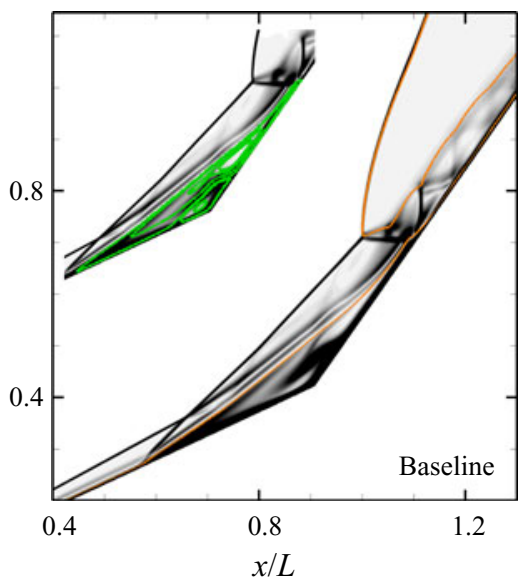

Figure 4. Contours of the density gradient magnitude superimposed with streamlines and sonic lines: $(a)$ $0.33 R e,(b) 0.5 R e,(c) 0.75 R e$ and $(d)$ baseline.

develops along the first cone. Near the separation point, $C_{f}$ experiences a sudden decrease to a local minimum and then increases gradually. Downstream of the corner, $C_{f}$ drops again to two successive local minima. The one closer to the reattachment point cannot be seen in supersonic compression corner flows, which is caused by the impingement of the transmitted shock. Downstream of reattachment, the skin friction rises to the peak and decreases with oscillations due to the wave reflection in the supersonic jet. At $x / L \approx 1.5$, the local peak is caused by the expansion corner at the end of the second cone. For the $0.33 R e$ and $0.5 R e$ cases, $C_{f}$ remains negative inside the separation region. In contrast, $C_{f}$ becomes positive near the corner at larger Reynolds numbers, which indicates the presence of secondary separation.

The distributions of the surface pressure coefficient and Stanton number are plotted for different Reynolds numbers in figure 6. Open circles mark the separation and reattachment points. From bottom to top, the horizontal lines represent inviscid pressure coefficients on the first and second cones, respectively. Upstream of separation, $C_{p}$ remains constant. Near the separation point, $C_{p}$ begins to rise and enters a plateau region. Then, the pressure rises dramatically to its peak value near flow reattachment and decreases to approach the cone value determined by inviscid theory for the second cone. The peak 

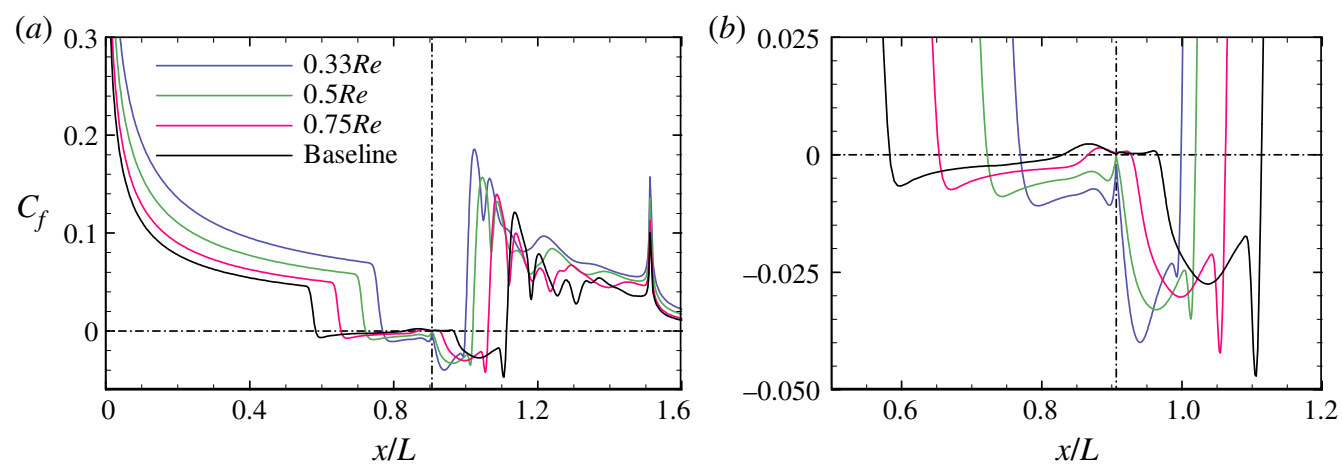

Figure 5. (a) Distributions of the skin friction coefficient for different Reynolds numbers with $(b)$ an enlarged view near the corner. Horizontal line, zero skin friction; vertical line, corner.
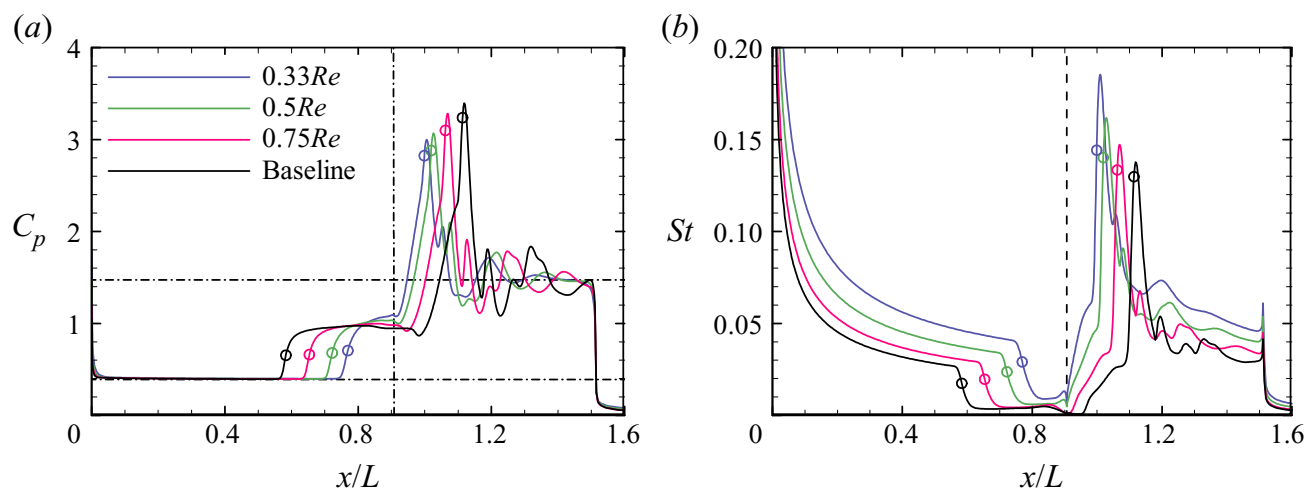

Figure 6. Distributions of ( $a$ ) the surface pressure coefficient and $(b)$ the surface Stanton number for different Reynolds numbers. Open circles, separation and reattachment points; horizontal lines, inviscid theory for the first and second cones; vertical line, corner.

value of $C_{p}$ increases with increasing Reynolds number. For all cases, a slight pressure 'dip' can be observed at the end of the pressure plateau. Such behaviour has been reported extensively for shock-wave-boundary-layer interactions over many canonical configurations (Shvedchenko 2009; Khraibut et al. 2017; Gai \& Khraibut 2019). In fact, the pressure 'dip' is the footprint of the low pressure in the vortex core of the primary bubble. For the highest Reynolds number, a second pressure plateau is seen near the corner corresponding to the secondary bubble (Korolev, Gajjar \& Ruban 2002). The surface heat flux behaves similarly to the skin friction in the incoming boundary layer. Stanton number $S t$ enters a valley region downstream of separation and increases to the peak value near the reattachment point, which decreases with increasing Reynolds number.

The behaviour of the pressure 'dip' is demonstrated in figure 7, which plots the distributions of the streamwise gradient of the surface pressure coefficient along the model surface for different Reynolds numbers. The figure also shows an enlarged view near the corner. Here, $s^{*}$ is the non-dimensional distance along the model surface measured from the vertex. The separation and reattachment points of the primary and secondary bubbles are indicated by open and filled circles, respectively. Downstream of the separation point, there is a nearly zero-pressure-gradient region corresponding to the pressure plateau. Downstream of the corner, there is a local minimum of the streamwise pressure 
(a)

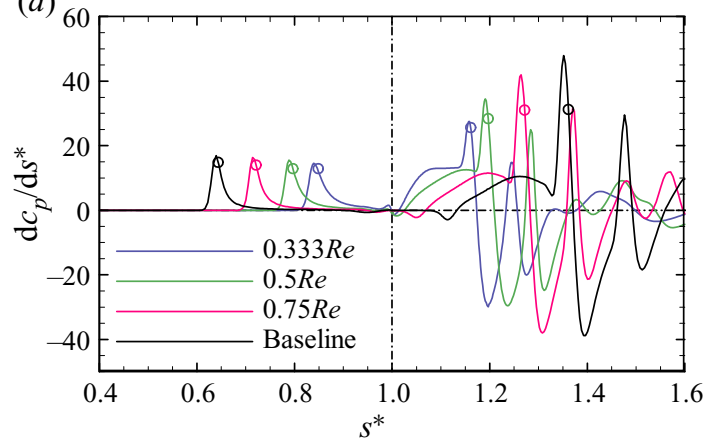

(b)

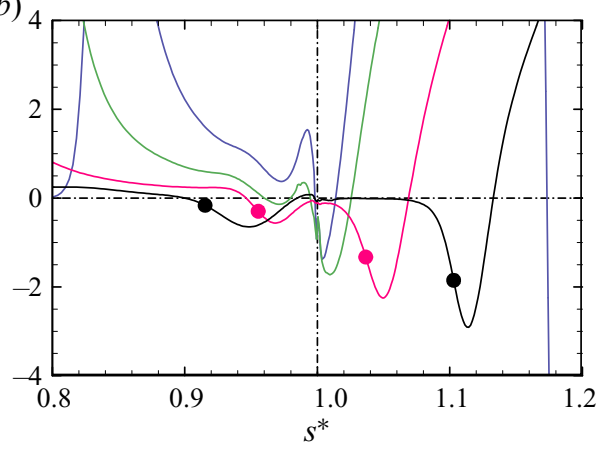

Figure 7. (a) Distributions of the streamwise gradient of the surface pressure coefficient along the model surface for different Reynolds numbers with $(b)$ an enlarged view near the corner. Open circles, separation and reattachment points of primary separation; filled circles, separation and reattachment points of secondary separation; vertical line, corner.

gradient induced by the pressure 'dip'. Its magnitude increases with increasing Reynolds number. Beyond a certain value, the reverse flow boundary layer developing from the primary reattachment point cannot resist the adverse pressure gradient and separates into a secondary bubble. Inside the secondary separation region, another zero-pressure-gradient region can be observed for the baseline case.

Figure 8 compares the distributions of the surface pressure coefficient and Stanton number with the experimental data of Holden et al. (2003) for the 0.33Re, 0.5Re and baseline cases. Good agreement is obtained in terms of the size of the separation region and the peak values and locations of $C_{p}$ and $S t$, except that the heat flux on the first cone is slightly overestimated. As previously mentioned, this is caused by the frozen vibrational energy in the free stream and its weak accommodation at the wall.

\subsection{Comparison with triple-deck theory}

Shock-induced separation is essentially a viscous-inviscid interaction problem. Neiland (1969) and Stewartson \& Williams (1969) proposed that the interaction region consisted of three tiers: an inviscid, irrotational upper deck, an inviscid, rotational middle deck and a viscous lower deck and found that the flow in the lower deck was governed by the incompressible boundary-layer equations after scaling.

The classical triple-deck theory has been applied to supersonic flow over a two-dimensional compression corner with moderate to high wall temperatures (Rizzetta, Burggraf \& Jenson 1978; Smith \& Khorrami 1991; Korolev et al. 2002). Its classical triple-deck solution depends on a scaled ramp angle $\alpha^{*}$ only. As $\alpha^{*}$ is increased from a small value to approximately 1.57, incipient separation occurs (Ruban 1976; Rizzetta et al. 1978; Ruban 1978). The length of the separation region continuously increases with $\alpha^{*}$ accompanied by the formation of a pressure plateau. Beyond a critical value of 4.5 , secondary separation is formed beneath the primary bubble (Smith \& Khorrami 1991; Korolev et al. 2002). Further increasing $\alpha^{*}$ leads to fragmentation of the primary bubble and eventually unsteadiness. Similar behaviours have been reported from two-dimensional CFD simulations (Shvedchenko 2009; Gai \& Khraibut 2019); however, the values of $\alpha^{*}$ for incipient and secondary separation moderately depend on the wall temperature ratio. A cold wall postpones incipient separation and facilitates secondary separation, as shown by Egorov, Neiland \& Shvedchenko (2011) and Hao et al. (2021). For supersonic 

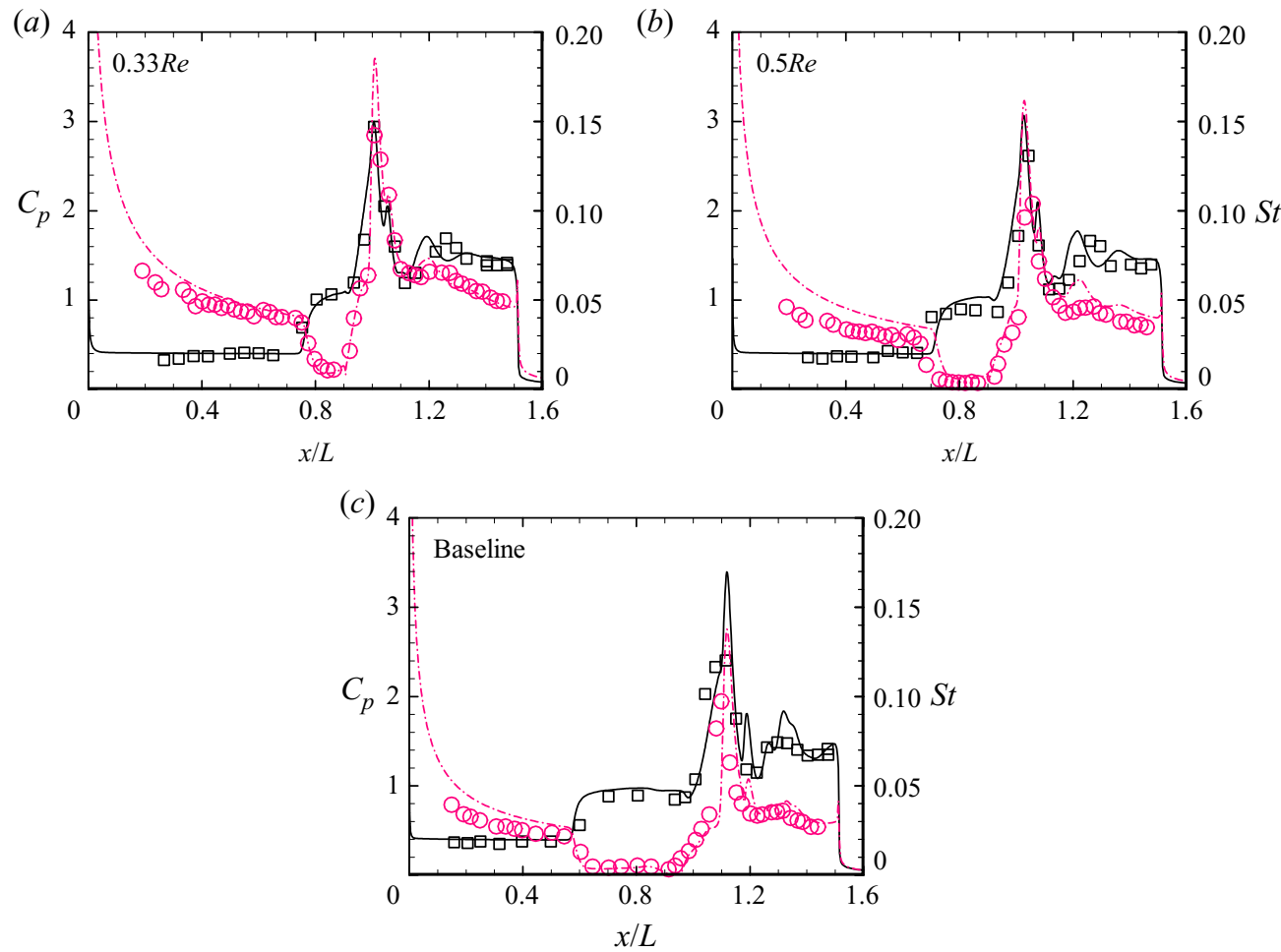

Figure 8. Comparison between the numerical and experimental distributions of the surface pressure coefficient and Stanton number: (a) 0.33Re, (b) 0.5Re and (c) baseline. Symbols, experimental data; solid line, pressure coefficient; dash-dotted line, Stanton number.

compression corner flows, the critical $\alpha^{*}$ for secondary separation ranges from 3.5 to 5 (Gai \& Khraibut 2019; Hao et al. 2021). The lower and upper boundaries correspond to the cold- and adiabatic-wall conditions, respectively. Note that the classical triple-deck theory implicitly requires that the wall temperature should be of the same order as the recovery temperature (Cheng 1993). The influence of wall cooling was considered by Neiland (1973), Neiland \& Sokolov (1975), Brown, Cheng \& Lee (1990), Kerimbekov, Ruban \& Walker (1994) and Cassel, Ruban \& Walker (1996). In the extended triple-deck formulation of Kerimbekov et al. (1994), the solution of a compression corner flow also depends on the level of wall cooling and the Mach number distribution across the boundary layer upstream of interaction. In this study, the double-cone flows are examined using the classical triple-deck scaling for supersonic flow. Comparison with the strong wall cooling theories will be made in a future study. Also note that hypersonic viscous interaction (Neiland 1970; Brown, Stewartson \& Williams, 1975; Brown et al. 1990; Smith \& Khorrami 1994) is not considered here.

Here, a new coordinate system is constructed with the $x_{1}$ axis along the first cone and the origin at the vertex, as shown in figure 1. Obviously, the body radius at the corner is of the same order of magnitude as L. According to Huang \& Inger (1983), the only axisymmetric effect is that on the incoming boundary layer as considered by the Mangler transformation, and the triple-deck equations remain the same as those for two-dimensional flows. If the angle of the first cone is small enough, the three-dimensional relieving effect enters the upper deck and reduces the pressure rise due to flow deflection, which can significantly 


\section{Three-dimensionality of hypersonic laminar flow}

postpone both incipient and secondary separation (Gittler \& Kluwick 1987). In addition, as pointed out by Kluwick, Gittler \& Bodonyi (1984), Huang \& Inger (1983) only considered a linearized solution of the triple-deck equations, which resulted in a non-smooth pressure distribution at the corner.

The scaled lower-deck variables relevant to this study are given as follows:

$$
\begin{gathered}
x^{*}=R e^{3 / 8} C^{-3 / 8} \lambda^{5 / 4}\left(M_{e}^{2}-1\right)^{3 / 8}\left(\frac{T_{w}}{T_{e}}\right)^{-3 / 2} \frac{x_{1}-L}{L}, \\
p^{*}=R e^{1 / 4} C^{-1 / 4} \lambda^{-1 / 2}\left(M_{e}^{2}-1\right)^{1 / 4} \frac{p-p_{e}}{\rho_{e} u_{e}^{2}}, \\
\alpha^{*}=\operatorname{Re}^{1 / 4} C^{-1 / 4} \lambda^{-1 / 2}\left(M_{e}^{2}-1\right)^{-1 / 4} \alpha, \\
\operatorname{Re}=\frac{\rho_{e} u_{e} L}{\mu_{e}}, \quad C=\frac{\mu_{w} T_{e}}{\mu_{e} T_{w}},
\end{gathered}
$$

where $x^{*}$ is the scaled distance measured from the corner, Re is the Reynolds number based on the flow properties at the edge of the incoming boundary layer (denoted by the subscript $e$ ) and $L, C$ is the Chapman-Rubesin parameter, $\lambda$ is a value determined by the slope of the velocity profile at the wall in the incoming boundary layer, $\alpha$ is the deflection angle in radians and $p^{*}$ is the scaled pressure.

The flow properties at the edge of the incoming boundary layer are determined by solving the Taylor-Maccoll equations. For the Blasius boundary layer over a flat plate, $\lambda$ equals 0.3322 . Here, $\lambda$ should be multiplied by a factor of $\sqrt{ } 3$ according to the Mangler transformation for the boundary layer over the first cone.

For the four flow conditions under consideration, the values of $\alpha^{*}$ are 2.872, 3.178, 3.518 and 3.780. From the base-flow solutions (see figures 4 and 5), secondary separation occurs when $\alpha^{*}$ is between 3.178 and 3.518. The critical value is approximately 3.327 determined by additional simulations. As expected, this is close to the lower boundary of the critical $\alpha^{*}$ for secondary separation in supersonic compression corner flows because of the relatively low wall temperature ratio. Secondary separation in the double-cone flows considered by Tumuklu et al. (2018) occurs when $\alpha^{*}$ is between 3.696 and 4.090. Note that their wall temperature ratio $(0.142)$ is greater than the current value $(0.079)$, resulting in a slightly larger critical $\alpha^{*}$.

Figure 9 shows the scaled pressure distributions as a function of $x^{*}$ for different Reynolds numbers. The separation and reattachment points are also marked. Upstream of separation, $p^{*}$ is zero according to its definition. Near the separation point, the behaviour of $p^{*}$ is unaffected by the increase in $\alpha^{*}$, which is ruled by the free-interaction process (Chapman et al. 1958). Downstream of separation, the pressure plateau is approximately 1.8, which agrees well with the classical triple-deck scaling (Korolev et al. 2002). Near the reattachment point, the pressure increases dramatically. Note that the peak value is much larger than the corresponding $\alpha^{*}$. This is because the linearized theory used in the triple-deck theory to obtain the pressure-deflection relation in the upper deck no longer holds for the relatively high Mach number and deflection angle considered in this study. In fact, the linearized theory tends to underestimate the postshock pressure. The impingement of the transmitted shock further increases the pressure peak. As an outcome of the cold-wall effect, the extent of the separation region significantly exceeds the triple-deck result obtained for the same $\alpha^{*}$ (Rizzetta et al. 1978; Korolev et al. 2002). 


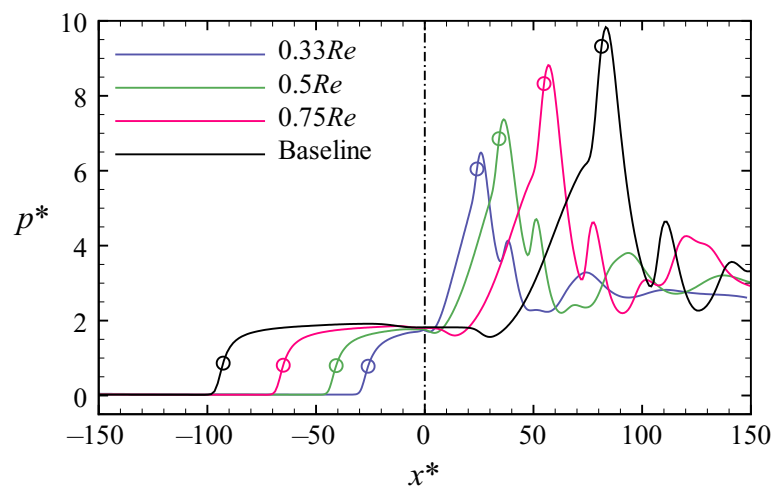

Figure 9. Distributions of scaled surface pressure for different Reynolds numbers. Open circles, separation and reattachment points.

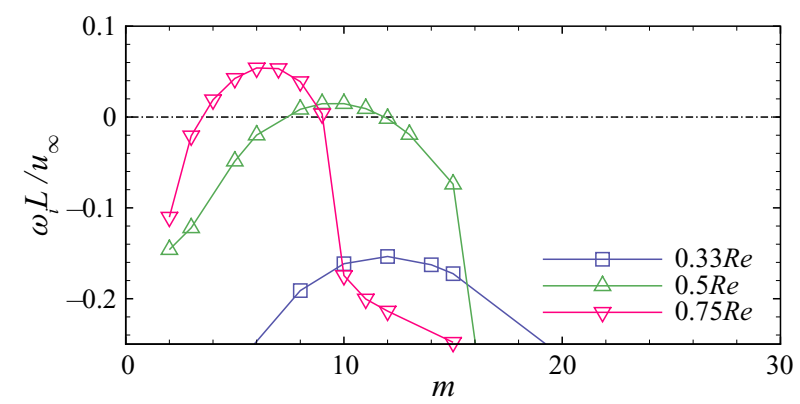

Figure 10. Variations in the growth rates of the least stable modes as a function of azimuthal wavenumber for different Reynolds numbers.

\subsection{Onset of global instability}

To determine the global stability of hypersonic double-cone flows with respect to azimuthally periodic perturbations, GSA is performed using the axisymmetric base flows over a wide range of azimuthal wavenumbers.

Figure 10 shows the growth rates of the least stable modes captured by the GSA for the $0.33 R e, 0.5 R e$ and $0.75 R e$ cases. For the lowest Reynolds number $\left(\alpha^{*}=2.872\right)$, the flow is stable with the least stable azimuthal wavenumber of 12 . For the $0.5 R e$ case $\left(\alpha^{*}=3.178\right)$, an unstable mode can be observed with $m=10$. As the Reynolds number is further increased, the maximum growth rate increases as the corresponding wavenumber shifts to $m=6$. For these cases, the least stable modes are stationary. In other words, the magnitude of the perturbation added to the base flow grows monotonically with time until nonlinear effects set in. The flow becomes unstable immediately prior to the emergence of secondary separation, which is consistent with the behaviour of global instability in supersonic compression corner flows (Hao et al. 2021). Therefore, the criterion of the stability boundary in terms of $\alpha^{*}$ established for supersonic compression corner and double-wedge flows (Hao et al. 2021) can be directly applied to double-cone flows.

Figure 11 shows the contours of the azimuthal velocity perturbation $w^{\prime}$ and pressure perturbation $p^{\prime}$ corresponding to the least stable modes for the $0.33 R e, 0.5 R e$ and $0.75 R e$ cases. The shock locations and dividing streamlines are superimposed. It is confirmed that these modes are of the same nature. In contrast to supersonic compression corner 
(a)
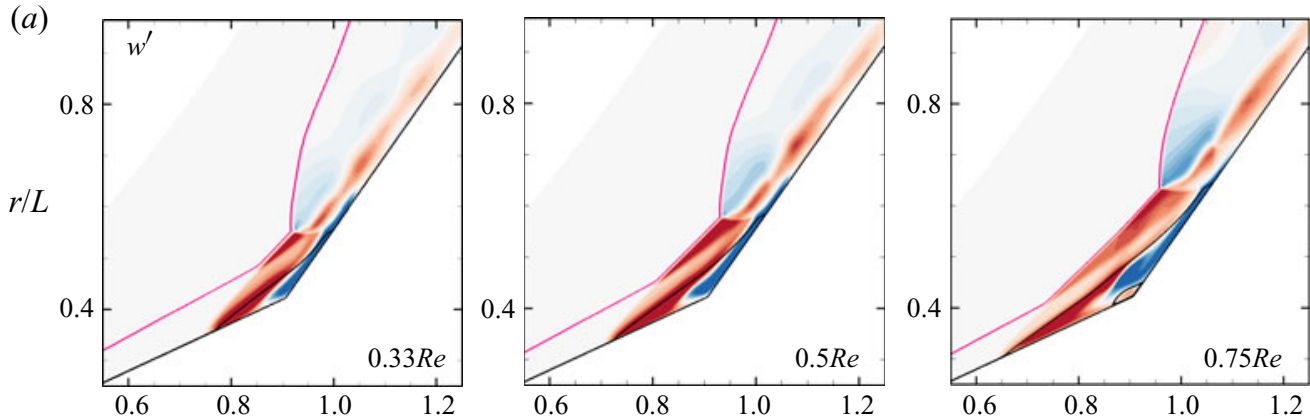

(b)
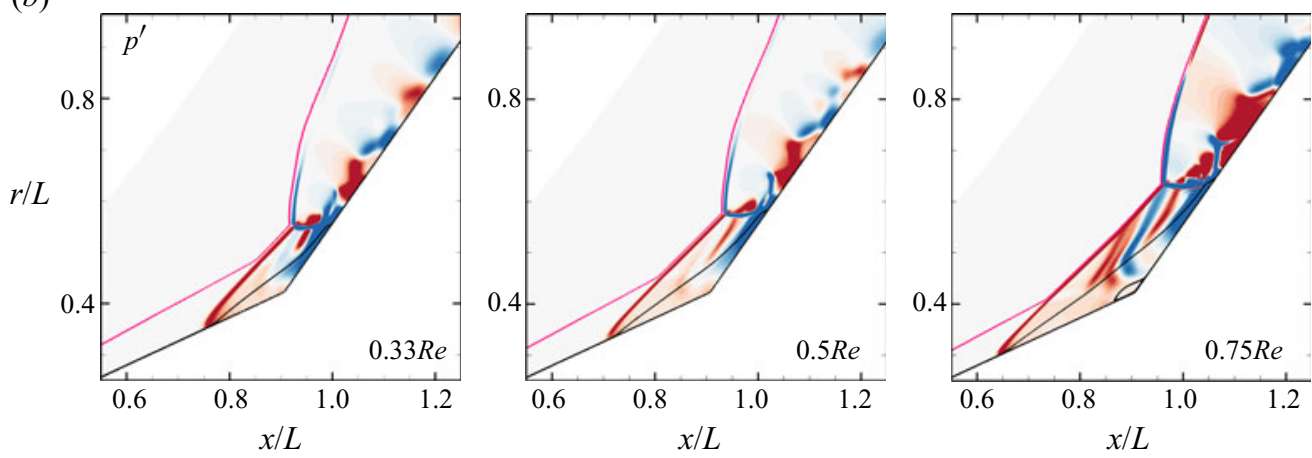

Figure 11. Contours of $(a)$ the azimuthal velocity perturbation and $(b)$ the pressure perturbation for different Reynolds numbers superimposed with shock locations and dividing streamlines. The contour levels are evenly spaced between \pm 0.5 of the maximum $\left|w^{\prime}\right|$ and between \pm 0.1 of the maximum $\left|p^{\prime}\right|$.

and double-wedge flows (Sidharth et al. 2018; Cao et al. 2021; Hao et al. 2021), $w^{\prime}$ is not confined in the separation region but also distributed in the inviscid regions behind the separation and curved shocks and in the supersonic jet. This may be attributed to the complexity of the shock interaction in the double-cone flows. The pressure perturbations further highlight the strong coupling between the separation region, separation and curved shocks and wave reflection in the supersonic jet. Similar observations were made by Tumuklu et al. (2018). However, they only conducted axisymmetric GSA and thus found no unstable modes. As the Reynolds number is increased, the pressure perturbation in the supersonic jet penetrates the subsonic region bounded by the curved shock and slip line.

For the highest Reynolds number, the flow is strongly destabilized. The growth rates and angular frequencies of the most unstable modes are plotted in figure 12 as a function of $m$. Note that mode 3 belongs to the same family as those shown in figure 10. The most unstable azimuthal wavenumber of mode 3 further decreases to 5. Meanwhile, a stationary mode (mode 1) and an oscillating mode (mode 2 ) occur with the growth rates peaking at $m=33$ and 30, respectively. The frequency of mode 2 at $m=30$ is approximately $f=$ $4.8 \mathrm{kHz}\left(f L / u_{\infty}=0.18\right)$, which belongs to the low-frequency regime. Modes 4 and 5 are also oscillating modes with even lower frequencies; however, these modes should be trivial due to the low growth rates.

The azimuthal velocity perturbation and pressure perturbation contours are shown in figure 13 for modes 1-3 at $m=33,30$ and 5. Modes 1-3 structurally resemble the dominant stationary and oscillating unstable modes found in supersonic double-wedge and compression corner flows (Sidharth et al. 2018; Hao et al. 2021), especially in the separation region. These unstable modes also originate from the separated flow; however, 

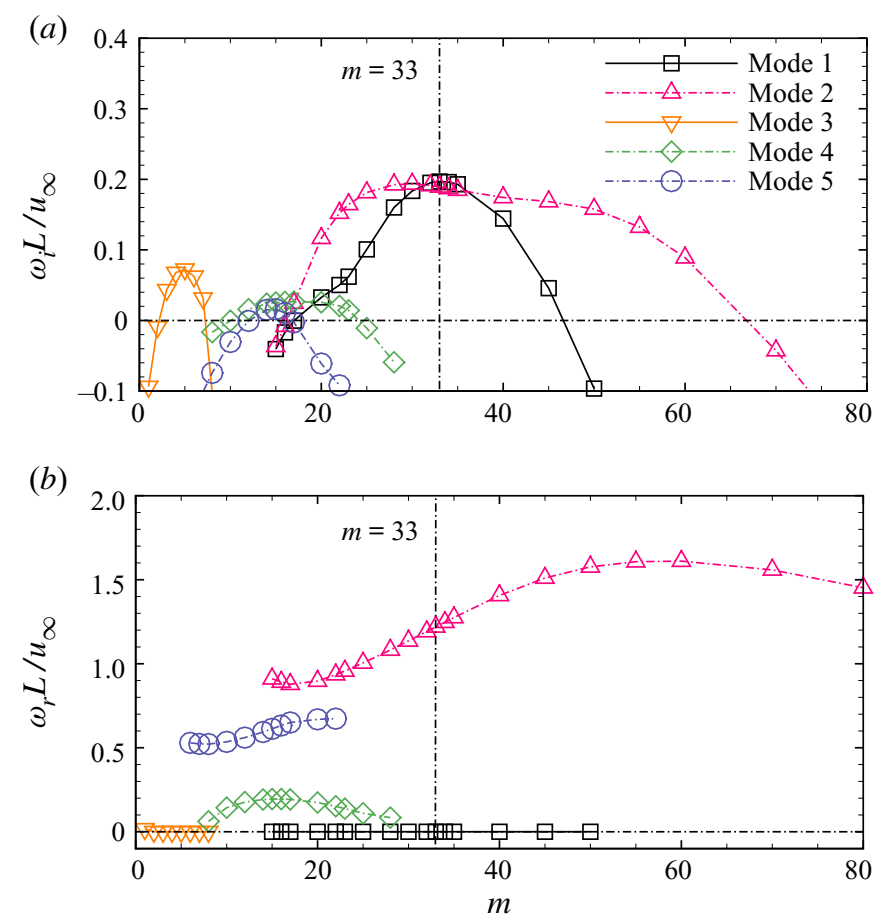

Figure 12. Variations in $(a)$ the growth rates and $(b)$ the angular frequencies of the most unstable modes as a function of azimuthal wavenumber for the baseline case.

they can be decorated by a particular type of shock interaction. Note that only Edney's type VI interaction was considered by Sidharth et al. (2018) and Hao et al. (2021). Here, $w^{\prime}$ and $p^{\prime}$ are also distributed in the supersonic jet with alternating signs in accordance with the wave reflection therein. The eigenfunctions of mode 3 are similar to those shown in figure 11 but characterized by a closer link between the supersonic jet and curved shock. Interestingly, modes 1 and 3 are absent in the secondary bubble in contrast to mode 2 .

\subsection{Direct numerical simulations}

In this section, DNS are performed to verify the GSA results and reveal the three-dimensional flow structures by solving the complete Navier-Stokes equations (3.1). Here, only the supercritical baseline case (i.e. run 35) is considered, which supports two stationary and three oscillating unstable modes.

The computational grid is generated by rotating the medium grid $(750 \times 350)$ around the axis over an angle of $72^{\circ}$ to make the DNS affordable. Hence, the azimuthal wavenumbers that can be numerically resolved must be integer multiples of 5 . This azimuthal domain extent is chosen because the growth rates of mode 1 at $m=30$ and 35 are close to the maximum value (see figure 12a). In addition, the growth rates of modes 2 and 3 peak at $m=30$ and 5, respectively. Two hundred grid cells are used in the azimuthal direction, which achieves a grid size comparable to that in the streamwise direction near the peak heating. Part of the grid upstream of separation is cut off to remove the axial singularity. The inlet boundary conditions are specified using the axisymmetric profile at the same streamwise location. Periodic boundary conditions are applied to the azimuthal boundaries. The initial flow field is constructed by duplicating the base flow 
(a)
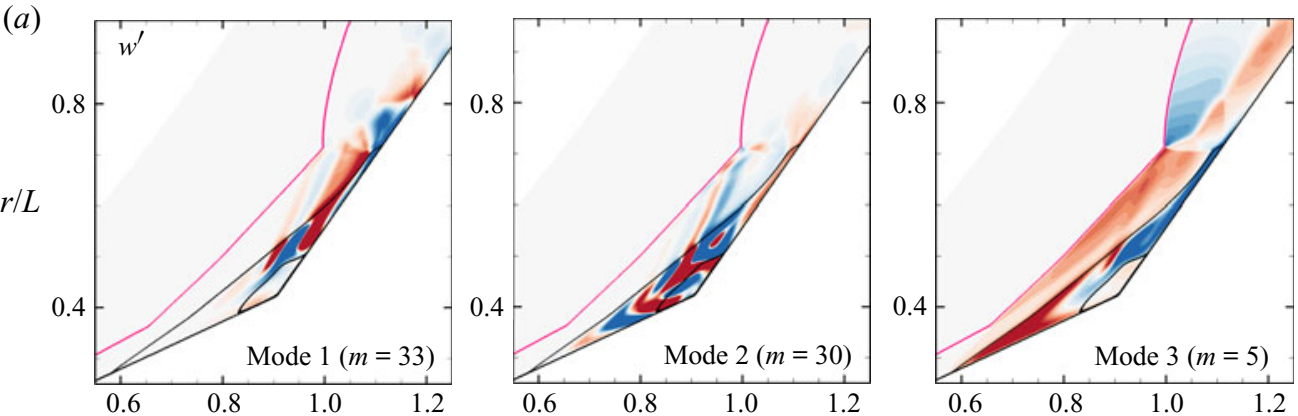

(b)
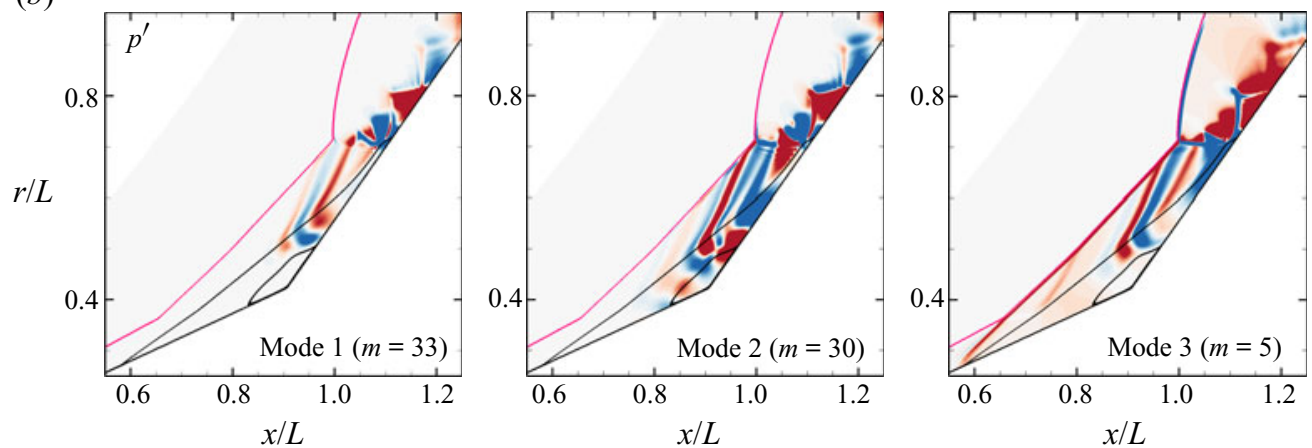

Figure 13. Contours of $(a)$ the azimuthal velocity perturbation and $(b)$ the pressure perturbation for modes 1-3 at $m=33,30$ and 5 for the baseline case superimposed with shock locations and dividing streamlines. The contour levels are evenly spaced between \pm 0.5 of the maximum $\left|w^{\prime}\right|$ and between \pm 0.1 of the maximum $\left|p^{\prime}\right|$.

in the azimuthal direction. Note that no external or internal disturbances are introduced. Therefore, global instability is expected to arise from the numerical round-off error.

The three-dimensional simulation is performed using PHAROS with the same numerical schemes as described in $\$ 3.2$, except that a second-order implicit scheme (Peterson 2011) is utilized for time marching. The physical time step is set to $20 \mathrm{~ns}$. An independence study verified that a smaller time step did not change the flow evolution. The simulation is run for a total physical time $t=14 \mathrm{~ms}\left(t u_{\infty} / L=373.8\right)$ to ensure a quasi-steady state and to fully resolve the low-frequency unsteadiness. The sampling frequency is $0.5 \mathrm{MHz}$ for spectral analysis.

To quantitatively characterize the evolution of global instability, the root mean square of the azimuthal velocity at a given streamwise station is defined by

$$
\sigma_{w}=\sqrt{\frac{1}{N_{r} N_{\phi}} \sum_{j=1}^{N_{r}} \sum_{k=1}^{N_{\phi}}\left(\frac{w}{u_{\infty}}\right)^{2}},
$$

where $N_{r}$ and $N_{\phi}$ are the numbers of grid cells in the radial and azimuthal directions, respectively. The temporal history of $\sigma_{w}$ at $x / L=0.98$ near the corner is shown in figure 14 . After the initialization, $\sigma_{w}$ experiences exponential growth until $t u_{\infty} / L \approx 70$ and nonlinear saturation. After $t u_{\infty} / L \approx 240$, a quasi-steady state is established. The growth rate of $\sigma_{w}$ in the linear stage agrees well with that of mode 1 at $m=30$ represented by the dashed-dotted line. 


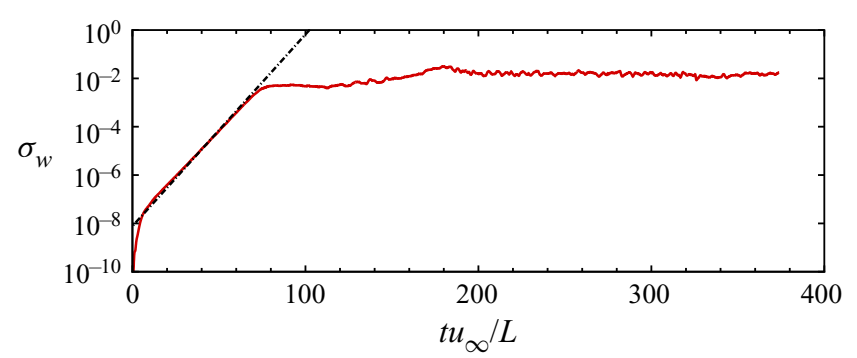

Figure 14. Temporal history of the root mean square of the azimuthal velocity at $x / L=0.98$. The slope of the dashed-dotted line equals the growth rate of mode 1 at $m=30$.

(a)

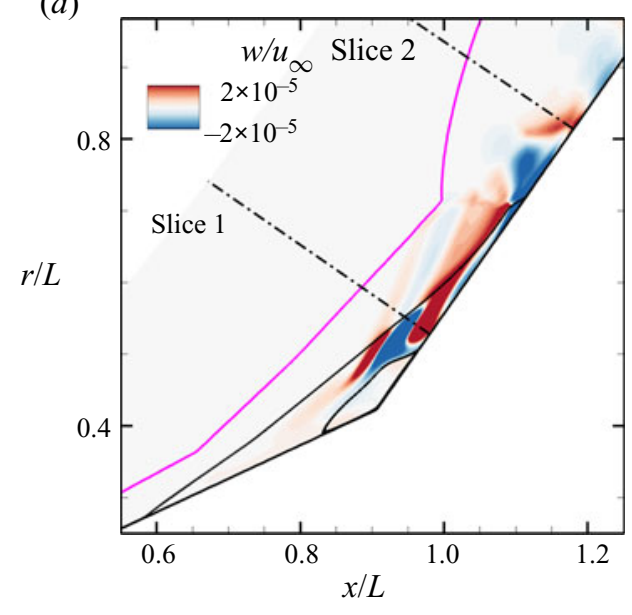

(b)
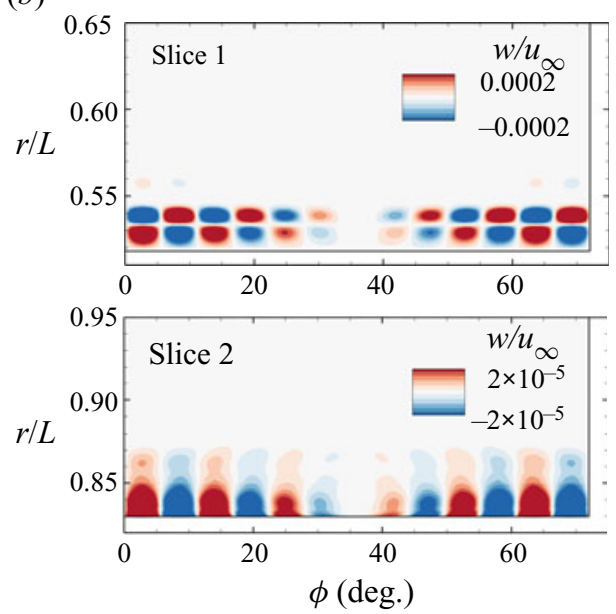

Figure 15. Contours of the azimuthal velocity in $(a)$ the $x-r$ plane at $\phi=42.5^{\circ}$ and $(b)$ two wall-normal slices extracted at $x / L=0.98$ and 1.20 at $t u_{\infty} / L=53.4$.

Figure 15(a) shows the contour of the azimuthal velocity in a certain $x-r$ plane $\left(\phi=42.5^{\circ}\right)$ at $t u_{\infty} / L=53.4$ in the mid-linear stage. The spatial structure is almost identical to that of mode 1 captured by the GSA (see figure 13a), which confirms that mode 1 dominates the linear growth of the three-dimensional perturbations. The contours of the azimuthal velocity are also plotted in two wall-normal slices extracted at $x / L=0.98$ and 1.20 in figure $15(b)$. Note that slices 1 and 2 are located in the separation bubble and downstream of reattachment, respectively. An azimuthal periodicity with $m=30$ can be seen.

Figure 16 shows the temporal history of the azimuthally averaged surface Stanton number at $x / L=1.10$ near flow reattachment. The heat flux remains constant at the beginning and oscillates in the late linear stage, which marks the emergence of mode 2 . As will be seen later, the steep increase in $S t$ at $t u_{\infty} / L \approx 170$ is caused by the enlargement of the separation region. A fast Fourier transformation is carried out from $t u_{\infty} / L=0$ to 80.1 with the resulting power spectral density (PSD) plotted in figure 17(a). The dominant non-dimensional frequency is consistent with that of mode 2 at $m=30$. The PSD of the surface Stanton number signal in the quasi-steady stage from $t u_{\infty} / L=240.3$ to 373.8 is presented in figure $17(b)$, which exhibits a broad-band feature also centred at $f L / u_{\infty} \approx 0.18$. 


\section{Three-dimensionality of hypersonic laminar flow}

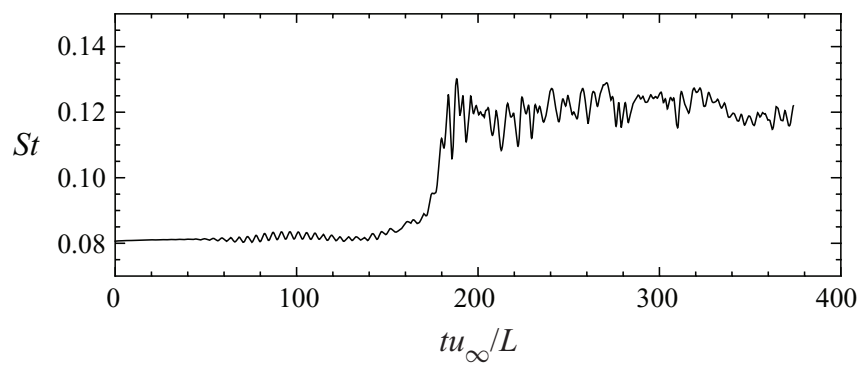

Figure 16. Temporal history of the azimuthally averaged surface Stanton number at $x / L=1.10$.

(a)

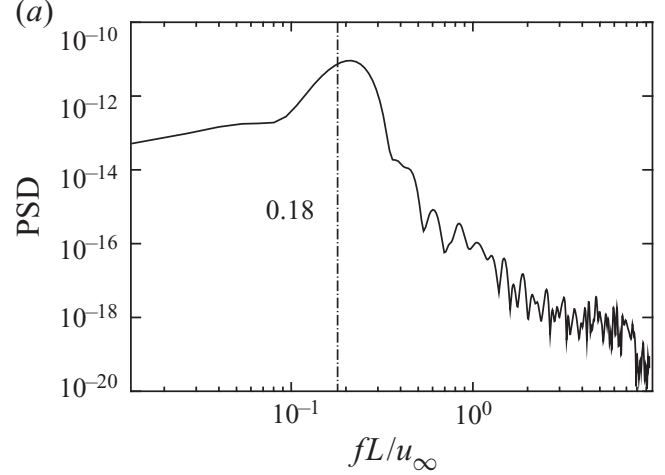

(b)

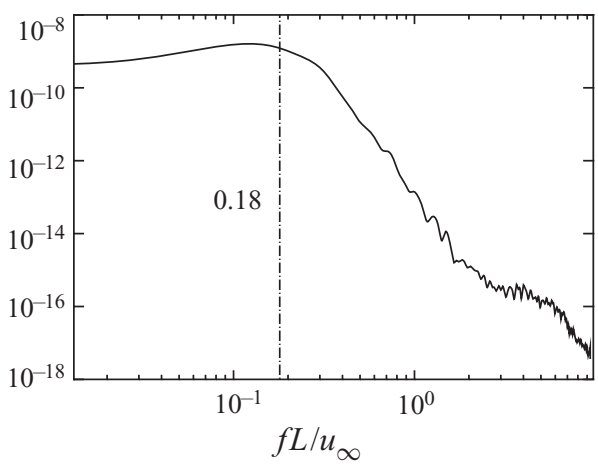

Figure 17. Power spectral density of the azimuthally averaged surface Stanton number signal at $x / L=1.10$ in two time intervals: $(a)$ from $t u_{\infty} / L=0$ to 80.1 and $(b)$ from $t u_{\infty} / L=240.3$ to 373.8 .

Figure 18 presents the contours of the surface Stanton number at different time instants. Isolines of $C_{f}=0$ are also plotted to indicate the separation regions. The separation and reattachment points obtained from the base-flow solution are marked by arrows. At $t u_{\infty} / L=0$, the contour is axisymmetric. At $t u_{\infty} / L=77.4$, the reattachment line of the primary bubble and the separation and reattachment lines of the secondary bubble become corrugated, accompanied by slight azimuthal variations in the heat flux. After $t u_{\infty} / L=154.9$, the secondary bubble is strongly distorted and eventually breaks up into several small bubbles. The separation line of the primary bubble remains largely unaffected by the three-dimensionality of the flow field downstream, whereas the reattachment line exhibits a zigzag pattern. Similar features were reported by Cao et al. (2021) in their DNS of hypersonic compression corner flows.

In contrast to the compression corner flows, no streamwise streaks that can persist for some distance are found downstream of reattachment. Instead, the narrow annular region of the maximum heat flux fragments into azimuthally periodic peaks and valleys. Meanwhile, the size of the separation region slightly increases compared to the axisymmetric value between $t u_{\infty} / L=154.9$ and 240.3, which explains the sudden increase in St shown in figure 16.

To closely examine the three-dimensional flow structures near flow reattachment, figure 19(a) shows an enlarged view of the surface Stanton number contour superimposed with skin friction lines at $t u_{\infty} / L=293$.7. Two wall-normal slices are extracted at $x / L=1.06$ and 1.16. The contours of the streamwise velocity in the two slices superimposed with in-plane streamlines are shown in figure $19(b)$. Note that the white lines indicate the 

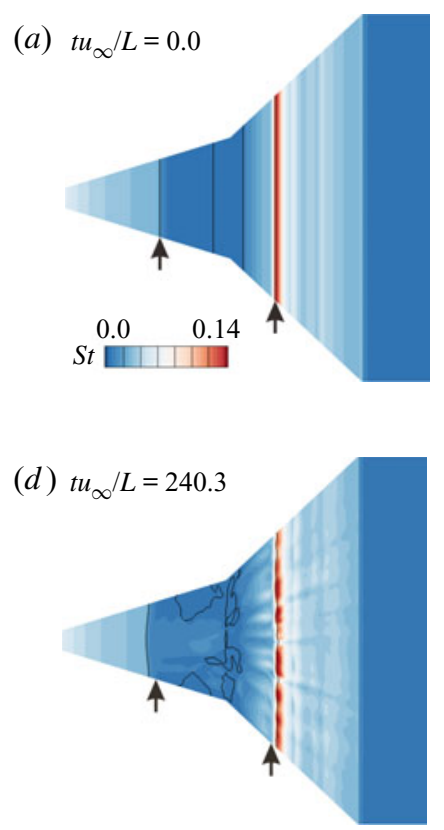

(b) $t u_{\infty} / L=77.4$

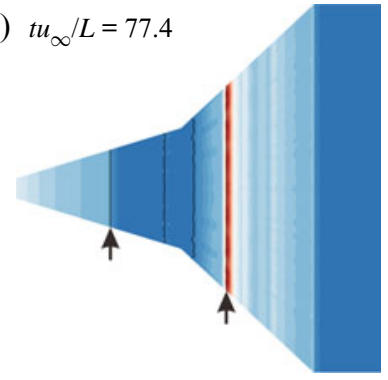

(e) $t u_{\infty} / L=293.7$

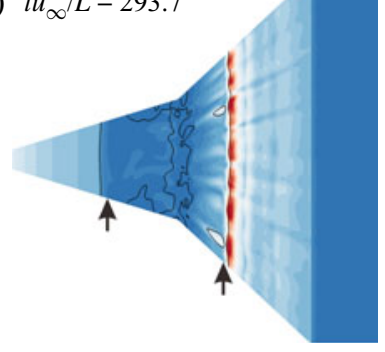

(c)

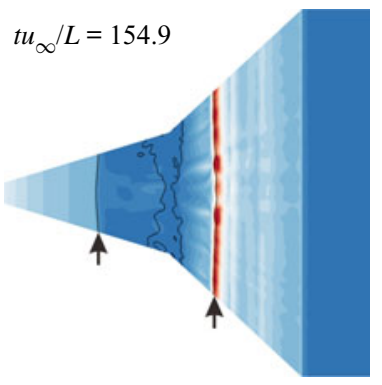

(f) $t u_{\infty} / L=347.1$

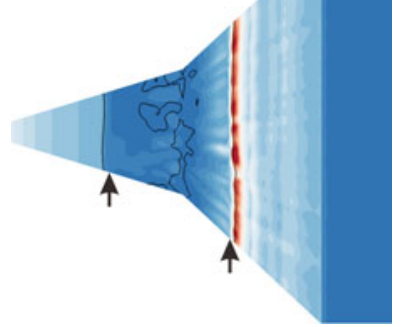

Figure 18. Contours of the surface Stanton number at (a) $t u_{\infty} / L=0,(b) t u_{\infty} / L=77.4$, (c) $t u_{\infty} / L=154.9,(d)$ $t u_{\infty} / L=240.3$, (e) $t u_{\infty} / L=293.7$ and $(f) t u_{\infty} / L=347.1$. Black solid lines, isolines of $C_{f}=0$; arrows, locations of the separation and reattachment points of the primary bubble obtained from the base-flow solution.

(a)

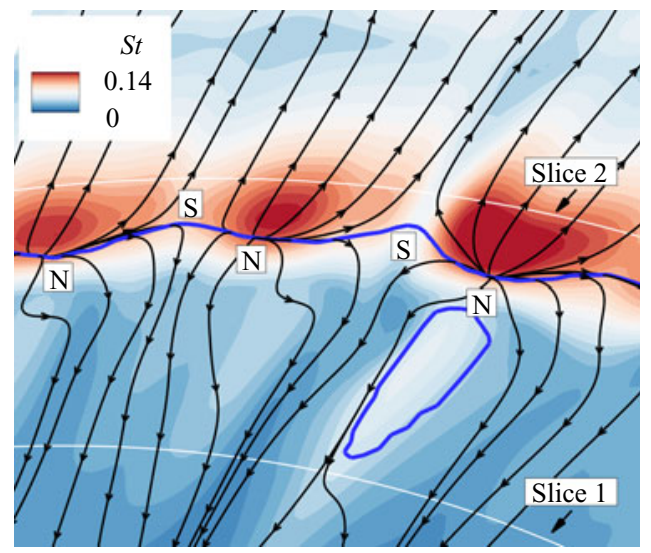

(b)

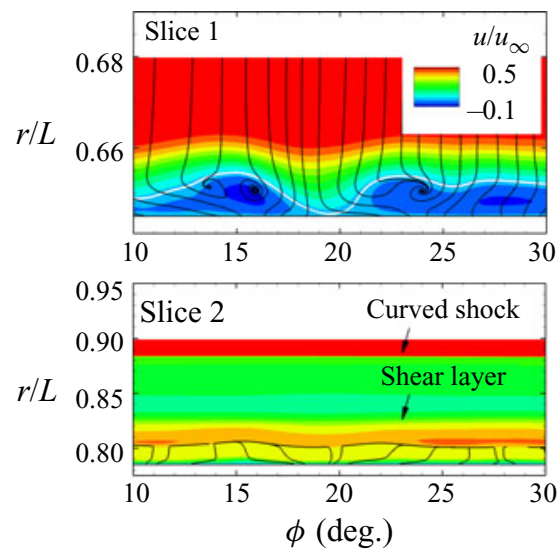

Figure 19. (a) Enlarged view of the contour of the surface Stanton number superimposed with skin friction lines. (b) Contours of the streamwise velocity in two wall-normal slices extracted at $x / L=1.06$ and 1.16 superimposed with in-plane streamlines at $t u_{\infty} / L=293.7$.

locations of the slices and zero streamwise velocity. In slice 1 located upstream of reattachment and downstream of the corner, the shear layer of the separation region is distorted by the counter-rotating streamwise vortical structures that are confined in the reversed flow region. The corrugated shear layer reattaches on the second cone to form a zigzag reattachment line with a series of nodes and saddle points shifted in the streamwise direction. The heat flux peaks are located downstream of the nodes, whereas 
(a)

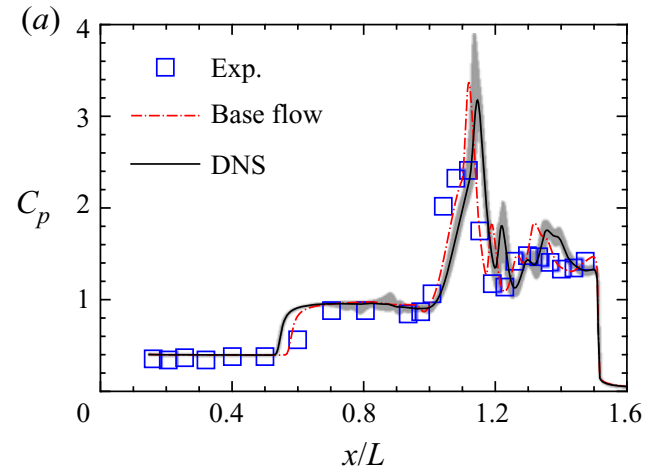

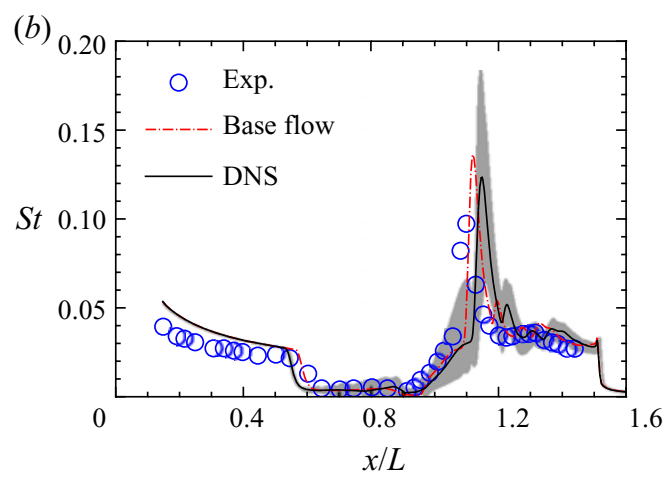

Figure 20. Comparison between the azimuthally averaged distributions of $(a)$ the surface pressure coefficient and $(b)$ the Stanton number at $t u_{\infty} / L=293.7$ and the experimental and base-flow data. The grey-shaded envelope represents the range of azimuthal variations.

the heat flux valleys are associated with the saddle points. In slice 2 located downstream of reattachment, another corrugated shear layer can be seen, emanating from the triple point (see figure 4). There are no streamwise vortices in the supersonic jet and reattaching boundary layer, which explains the absence of streamwise heat flux streaks downstream of reattachment.

The azimuthally averaged distributions of the surface pressure coefficient and Stanton number at $t u_{\infty} / L=293.7$ are plotted in figure 20 with the grey-shaded envelope bounded by the maximum and minimum values in the azimuthal direction. In general, the averaged DNS results agree well with the experimental and base-flow data. However, strong azimuthal variations in the heat flux exist, especially near flow reattachment, where the relative difference can be as high as $50 \%$ compared with the mean value.

\section{Conclusions}

The hypersonic laminar flow over a canonical $25^{\circ}-55^{\circ}$ double cone is investigated using CFD and GSA with varying unit Reynolds numbers. For all cases, no self-sustained inviscid unsteadiness is found, which is consistent with the unsteadiness boundary proposed by Hornung et al. (2021). The axisymmetric CFD reveals that the separation region is always terminated by the transmitted shock emanating from the interaction between the oblique and curved shocks induced by the first and second cones. The behaviours of skin friction, surface pressure and surface heat flux within the separation region are similar to those found in supersonic shock-induced separated flow over many canonical configurations. Secondary separation occurs beneath the primary bubble when the Reynolds number is greater than a critical value. The numerical predictions of the surface pressure and heat flux agree well with the experimental measurements. The numerical data are interpreted using the classical triple-deck scaling with the axisymmetric effect accounted for by the Mangler transformation. The universal values of the scaled separation and plateau pressures and the critical value of the scaled deflection angle for the emergence of the secondary separation also hold for double-cone flows. Note that the free-stream Mach number and wall temperature ratio are fixed in this study. The entire parameter space remains to be explored.

At relatively low Reynolds numbers, a stationary mode is captured by the GSA, whose growth rate increases as the Reynolds number is increased. Beyond a critical value, the mode becomes unstable with respect to azimuthally periodic perturbations. Meanwhile, 


\section{J. Hao, J. Fan, S. Cao and C.-Y. Wen}

the azimuthal wavenumber corresponding to the peak growth rate decreases. This mode is confined in the separation region and widely distributed behind the separation and curved shocks and within the supersonic jet. The onset of global instability occurs immediately prior to the emergence of secondary separation, which indicates that the instability criterion established for supersonic double-wedge and compression corner flows can be directly applied to double-cone flows. As the Reynolds number is further increased, the unstable mode continues to be destabilized. Meanwhile, an additional stationary and a low-frequency oscillating mode appear and become dominant at much larger azimuthal wavenumbers. Although generally resembling those found in supersonic double-wedge and compression corner flows, the structures of these modes are further complicated by the strong shock interaction in the double-cone flows.

The GSA results are verified by DNS initialized from the base-flow solution for the baseline case with no external or internal disturbances. The DNS reveal that the double-cone flow is highly three-dimensional and unsteady with strong variations in the surface heat flux near flow reattachment. It is indicated that axisymmetric laminar simulations would be questionable under flow conditions that are far beyond the global stability boundary. However, the GSA provides little information on convective instabilities that may happen locally in the flow field, e.g. the first and second modes (Mack 1975; Smith 1989; Smith \& Brown 1990; Fedorov 2011; Butler \& Laurence 2021) and vortical disturbances (Paredes, Choudhari \& Li 2016; Dwivedi et al. 2019). Potential laminar-turbulent transition induced by global and convective instabilities in hypersonic double-cone flows will be addressed in a future study.

Funding. This work is supported by the Hong Kong Research Grants Council (no. 15206519 and no. 25203721) and the National Natural Science Foundation of China (no. 12102377).

Declaration of interests. The authors report no conflict of interest.
Author ORCIDs.
() Jiaao Hao https://orcid.org/0000-0002-8571-4728;
Shibin Cao https://orcid.org/0000-0001-9579-0276;
(D) Chih-Yung Wen https://orcid.org/0000-0002-1181-8786.

\section{REFERENCES}

BABINSKY, H. \& HARVEY, J.K. 2011 Shock Wave-Boundary-Layer Interactions. Cambridge University Press.

Brown, S.N., Cheng, H.K. \& LeE, C.J. 1990 Inviscid-viscous interaction on triple-deck scales in a hypersonic flow with strong wall cooling. J. Fluid Mech. 220, 309-337.

Brown, S.N., Stewartson, K. \& Williams, P.G. 1975 Hypersonic self-induced separation. Phys. Fluids 18, 633-639.

BUtLER, C.S. \& LAURENCE, S.J. 2021 Interaction of second-mode disturbances with an incipiently separated compression-corner flow. J. Fluid Mech. 913, R4.

CaO, S., Hao, J., Klioutchnikov, I., Olivier, H. \& Wen, C.Y. 2021 Unsteady effects in a hypersonic compression ramp flow with laminar separation. J. Fluid Mech. 912, A3.

CAssel, K.W., Ruban, A.I. \& Walker, J.D.A. 1996 The influence of wall cooling on hypersonic boundary-layer separation and stability. J. Fluid Mech. 321, 189-216.

Chapman, D.R., KUehn, D.M. \& LARSON, H.K. 1958 Investigation of separated flows in supersonic and subsonic streams with emphasis on the effect of transition. NACA Tech. Rep. 1356.

Cheng, H.K. 1993 Perspectives on hypersonic viscous flow research. Annu. Rev. Fluid Mech. 25, 455-484.

Druguet, M.-C., CANDleR, G. \& Nompelis, V.I. 2005 Effect of numerics on Navier-Stokes computations of hypersonic double-cone flows. AIAA J. 43 (3), 616-623.

Dwivedi, A., Sidharth, G.S., Nichols, J.W., Candler, G.V. \& Jovanovic, M.R. 2019 Reattachment streaks in hypersonic compression ramp flow: an input-output analysis. J. Fluid Mech. 880, 113-135.

EDNEY, B.E. 1968 Effects of shock impingement on the heat transfer around blunt bodies. AIAA J. 6 (1), $15-21$. 


\section{Three-dimensionality of hypersonic laminar flow}

Egorov, I.V., Neiland, V.Y. \& Shvedchenko, V.V. 2011 Three-dimensional flow structures at supersonic flow over the compression ramp. AIAA Paper 2011-0730.

Fedorov, A. 2011 Transition and stability of high-speed boundary layers. Annu. Rev. Fluid Mech. 43, 79-95.

GAi, S.L. \& Khraibut, A. 2019 Hypersonic compression corner flow with large separated regions. J. Fluid Mech. 877, 471-494.

Gaitonde, D.V., CAnupp, P.W. \& Holden, M.S. 2002 Heat transfer predictions in a laminar hypersonic viscous/inviscid interaction. J. Thermophys. Heat Transfer 16 (4), 481-489.

GitTler, P.H. \& KLUWICK, A. 1987 Triple-deck solutions for supersonic flows past flared cylinders. J. Fluid Mech. 179, 469-487.

HaO, J., CaO, S., Wen, C.-Y. \& Olivier, H. 2021 Occurrence of global instability in hypersonic compression corner flow. J. Fluid Mech. 919, A4.

HAO, J., WANG, J. \& LEE, C. 2016 Numerical study of hypersonic flows over reentry configurations with different chemical nonequilibrium models. Acta Astron. 126, 1-10.

HAO, J. \& WEN, C.-Y. $2018 a$ Numerical investigation of oxygen thermochemical nonequilibrium on high-enthalpy double-cone flows. Intl J. Heat Mass Transfer 127, 892-902.

HAO, J. \& WEN, C.-Y. $2018 b$ Effects of vibrational nonequilibrium on hypersonic shock-wave/laminar boundary-layer interactions. Intl Commun. Heat Mass Transfer 97, 136-142.

HAO, J. \& WEN, C. Y. 2020 Hypersonic flow over spherically blunted double cones. J. Fluid Mech. 896, A26.

Hendrickson, T., Kartha, A. \& CAndler, G.V. 2018 An improved Ducros sensor for the simulation of compressible flows with shocks. AIAA Paper 2018-3710.

Hildebrand, N., Dwivedi, A., Nichols, J.W., Jovanović, M.R. \& Candler, G.V. 2018 Simulation and stability analysis of oblique shock-wave/boundary-layer interactions at Mach 5.92. Phys. Rev. Fluids 3 (1), 013906.

Holden, M.S. \& WAdhams, T.P. 2003 A database of aerothermal measurements in hypersonic flow in 'building block' experiments for CFD validation. AIAA Paper 2003-1137.

Holden, M.S., Wadhams, T.P., Candler, G.V. \& Harvey, J.K. 2003 Measurements in regions of low density laminar shock wave/boundary layer interaction in hypervelocity flows and comparison with Navier-Stokes predictions. AIAA Paper 2003-1131.

Holden, M.S., Wadhams, T.P., Maclean, M. \& Dufrene, A. 2015 Experimental research and analysis in supersonic and hypervelocity flows in the LENS shock tunnels and expansion tunnel. AIAA Paper 2015-3660.

Hornung, H.G., Gollan, R.J. \& JACOBS, P.A. 2021 Unsteadiness boundaries in supersonic flow over double cones. J. Fluid Mech. 916, A5.

HUANG, M.-K. \& INGER, G.R. 1983 Application of the triple-deck theory of viscous-inviscid interaction to bodies of revolution. J. Fluid Mech. 129, 427-441.

Kerimbekov, R.M., Ruban, A.I. \& WAlker, J.D.A. 1994 Hypersonic boundary-layer separation on a cold wall. J. Fluid Mech. 274, 163-195.

Khraibut, A., GAi, S.L., Brown, L.M. \& NeELy, A.J. 2017 Laminar hypersonic leading edge separation - a numerical study. J. Fluid Mech. 821, 624-646.

Kluwick, A., GitTler, P. \& Bodonyi, R.J. 1984 Viscous-inviscid interactions on axisymmetric bodies of revolution in supersonic flow. J. Fluid Mech. 140, 281-301.

Knight, D., Longo, J., Drikakis, D., Gaitonde, D., Lani, A., Nompelis, I., Reimann, B. \& WALPOT, L. 2012 Assessment of CFD capability for prediction of hypersonic shock interactions. Prog. Aerosp. Sci. 48-49, 8-26.

Korolev, G.L., GajJar, J.B. \& Ruban, A.I. 2002 Once again on the supersonic flow separation near a corner. J. Fluid Mech. 463, 173-199.

MACCORMACK, R.W. 2014 Numerical Computation of Compressible and Viscous Flow. AIAA.

MACK, L.M. 1975 Linear stability theory and the problem of supersonic boundary-layer transition. AIAA J. 13 (3), 278-289.

MANI, A. 2012 Analysis and optimization of numerical sponge layers as a nonreflective boundary treatment. J. Comput. Phys. 231, 704-716.

NeILAND, V.YA. 1969 Theory of laminar boundary layer separation in supersonic flow. Fluid Dyn. 4 (4), 33-35.

NEILAND, V.YA. 1970 Propagation of perturbations upstream with interaction between a hypersonic flow and a boundary layer. Izv. Akad. Nauk SSSR Mekh. Zhidk. Gaza 5 (4), 40-49.

NEILAND, V.YA. 1973 Peculiarities of boundary-layer separation on a cooled body and its interaction with a hypersonic flow. Izv. Akad. Nauk SSSR Mekh. Zhidk. Gaza 6, 99-109. 


\section{J. Hao, J. Fan, S. Cao and C.-Y. Wen}

Neiland, V.YA. \& SoKOlOV, L.A. 1975 On the asymptotic theory of incipient separation in compression ramp hypersonic flow on cooled body for a weak hypersonic interaction regime. Uchen. Zap. TsAGI 6 (3), 25-34.

Nompelis, I., Candler, G.V. \& Holden, M.S. 2003 Effect of vibrational nonequilibrium on hypersonic double-cone experiments. AIAA J. 41 (11), 2162-2169.

Olejniczak, J., Wright, M.J. \& CANDler, G.V. 1997 Numerical study of inviscid shock interactions on double-wedge geometries. J. Fluid Mech. 352, 1-25.

Paredes, P., Choudhari, M.M. \& Li, F. 2016 Transition due to streamwise streaks in a supersonic flat plate boundary layer. Phys. Rev. Fluids 1, 083601.

Peterson, D.M. 2011 Simulations of injection, mixing, and combustion in supersonic flow using a hybrid RANS/LES approach. PhD dissertation, University of Minnesota.

Rizzetta, D.P., Burggraf, O.R. \& Jenson, R. 1978 Triple-deck solutions for viscous supersonic and hypersonic flow past corners. J. Fluid Mech. 89, 535-552.

RoBINET, J. 2007 Bifurcations in shock-wave/laminar-boundary-layer interaction: global instability approach. J. Fluid Mech. 579, 85-112.

RUBAN, A.I. 1976 A numerical method for solving the free-interaction problem. Uchen. zap. TsAGI 7 (2), 45-51.

RUBAN, A.I. 1978 Numerical solution of the local asymptotic problem of the unsteady separation of a laminar boundary layer in a supersonic flow. Zh. vychisl. Mat. mat. Fiz. 18 (5), 1253-1265.

ShVEDCHENKo, V.V. 2009 About the secondary separation at supersonic flow over a compression ramp. TsAGI Sci. J. 40 (5), 587-607.

Sidharth, G.S., Dwivedi, S., CAndler, A., \& Nichols, G.V. \& W, J. 2018 Onset of three-dimensionality in supersonic flow over a slender double wedge. Phys. Rev. Fluids 3, 093901.

Stewartson, K. \& Williams, P.G. 1969 Self-induced separation. Proc. R. Soc. A 312, 181-206.

SMith, F.T. 1989 On the first-mode instability in subsonic, supersonic or hypersonic boundary layers. J. Fluid Mech. 198, 127-153.

SMith, F.T. \& BROWN, S.N. 1990 The inviscid instability of a Blasius boundary layer at large values of the Mach number. J. Fluid Mech. 219, 499-518.

Smith, F.T. \& KhorRami, A.F. 1991 The interactive breakdown in supersonic ramp flow. J. Fluid Mech. 224, 197-215.

Smith, F.T. \& KhORRAmi, A.F. 1994 Hypersonic aerodynamics on thin bodies with interaction and upstream influence. J. Fluid Mech. 277, 85-108.

Sorensen, D., LehoucQ, R., YAng, C. \& MAschHoff, K. 1996 ARPACK software, version 2.3.

Theofilis, V. 2011 Global linear instability. Annu. Rev. Fluid Mech. 43, 319-352.

Theofilis, V., Hein, S. \& Dallmann, U. 2000 On the origins of unsteadiness and three-dimensionality in a laminar separation bubble. Phil. Trans. R. Soc. Lond. A: Math. Phys. Engng Sci. 358 (1777), 3229-3246.

Tumuklu, O., Theofilis, V. \& LeVIn, D.A. 2018 On the unsteadiness of shock-laminar boundary layer interactions of hypersonic flows over a double cone. Phys. Fluids 30, 106111.

VAN LEER, B. 1979 Towards the ultimate conservative difference scheme. J. Comput. Phys. 32 (1), $101-136$.

Wright, M.J., CAndler, G.V. \& Bose, D. 1998 Data-parallel line relaxation method for the Navier-Stokes equations. AIAA J. 36 (9), 1603-1609. 